\title{
ARTICLE OPEN \\ Activation of mTORC1 in subchondral bone preosteoblasts promotes osteoarthritis by stimulating bone sclerosis and secretion of CXCL12
}

\author{
Chuangxin Lin ${ }^{1,3}$, Liangliang Liu ${ }^{1}$, Chun Zeng ${ }^{1}$, Zhong-Kai Cui $\mathbb{D}^{2}$, Yuhui Chen ${ }^{1}$, Pinling Lai ${ }^{1,2}$, Hong Wang ${ }^{1}$, Yan Shao ${ }^{1}$, Haiyan Zhang ${ }^{1}$, \\ Rongkai Zhang ${ }^{1}$, Chang Zhao ${ }^{1}$, Hang Fang ${ }^{1}$, Daozhang Cai ${ }^{1}$ and Xiaochun Bai ${ }^{1,2}$
}

Increasing evidences show that aberrant subchondral bone remodeling plays an important role in the development of osteoarthritis (OA). However, how subchondral bone formation is activated and the mechanism by which increased subchondral bone turnover promotes cartilage degeneration during OA remains unclear. Here, we show that the mechanistic target of rapamycin complex 1 (mTORC1) pathway is activated in subchondral bone preosteoblasts (Osterix + ) from OA patients and mice. Constitutive activation of mTORC 1 in preosteoblasts by deletion of the mTORC1 upstream inhibitor, tuberous sclerosis 1 , induced aberrant subchondral bone formation, and sclerosis with little-to-no effects on articular cartilage integrity, but accelerated posttraumatic OA development in mice. In contrast, inhibition of mTORC1 in preosteoblasts by disruption of Raptor (mTORC1-specific component) reduced subchondral bone formation and cartilage degeneration, and attenuated post-traumatic OA in mice. Mechanistically, mTORC1 activation promoted preosteoblast expansion and Cxcl12 secretion, which induced subchondral bone remodeling and cartilage degeneration during $\mathrm{OA}$. A Cxcl12-neutralizing antibody reduced cartilage degeneration and alleviated $\mathrm{OA}$ in mice. Altogether, these findings demonstrate that mTORC1 activation in subchondral preosteoblasts is not sufficient to induce $\mathrm{OA}$, but can induce aberrant subchondral bone formation and secrete of $\mathrm{Cxcl} 12$ to accelerate disease progression following surgical destabilization of the joint. Pharmaceutical inhibition of the pathway presents a promising therapeutic approach for OA treatment.

Bone Research (2019)7:5; https://doi.org/10.1038/s41413-018-0041-8

\section{INTRODUCTION}

Osteoarthritis $(\mathrm{OA})$ is a highly prevalent and degenerative joint disorder which mainly affects the weight-bearing joints such as hips and knees, and is the leading cause of physical disability, ${ }^{1-3}$ presenting an enormous clinical and financial burden. However, no effective disease-modifying treatment for $O A$ is currently available until the disease reaches the end stage, necessitating joint replacement. ${ }^{4}$ Indeed, the development of OA disease affects all joint tissues, being characterized by progressive degeneration of articular cartilage, vascular invasion of the articular surface, subchondral bone remodeling, osteophyte formation, and synovial inflammation. ${ }^{5}$ Despite the identified risk factors, which include genetic predisposition, ${ }^{6}$ mechanical abnormality, ${ }^{7}$ age, $^{8}$ and obesity, ${ }^{9}$ the exact pathogenesis of OA remains unclear.

Recently, increasing evidences indicate that articular cartilage degeneration is related to aberrant subchondral bone turnover. ${ }^{1,10,11}$ Subchondral bone provides mechanical support for the overlying articular cartilage during the movement of joints and absorbs most of the mechanical force transmitted by diarthrodial joints. Relative to the slower turnover rate of articular cartilage, subchondral bone undergoes more rapid modeling and remodeling in response to changes of the mechanical environment. ${ }^{12}$ Indeed, homeostasis and integrity of articular cartilage rely on its biochemical and biomechanical interplay with subchondral bone and other joint tissues, ${ }^{13}$ and treatment targeting articular cartilage alone in $O A$ has proven to be insufficient to halt disease progression. In a situation of instability, such as occurring with ligament injury, ${ }^{14}$ excessive body weight, ${ }^{15}$ or weakening muscles related to aging, ${ }^{16}$ the mechanical loading on weight-bearing joints is dramatically increased, and changes in the subchondral bone microarchitecture may precede articular cartilage damage. Thus, changes in the subchondral bone have been suggested to predict the severity of cartilage damage in OA. ${ }^{17}$ However, the mechanism by which aberrant subchondral bone formation is induced and the interplay between increased subchondral bone turnover and articular cartilage degeneration during $\mathrm{OA}$ remains unclear.

Mechanistic target of rapamycin (mTOR) is a highly conserved Ser/Thr protein kinase that functions as a master regulator of cell growth, proliferation, survival, and metabolism in response to nutrients, growth factors, and stresses. ${ }^{18,19}$ mTOR forms two distinct functional complexes, mTOR complex 1 (mTORC1) and

\footnotetext{
${ }^{1}$ Department of Orthopedics, Academy of Orthopedics-Guangdong Province, The Third Affiliated Hospital of Southern Medical University, 510630 Guangzhou, China; ${ }^{2}$ Key Laboratory of Mental Health of the Ministry of Education, Department of Cell Biology, School of Basic Medical Sciences, Southern Medical University, 510515 Guangzhou, China and ${ }^{3}$ Department of Orthopedic Surgery, Shantou Central Hospital, Affiliated Shantou Hospital of Sun Yat-Sen University, 515041 Shantou, China

Correspondence: Daozhang Cai (cdz@smu.edu.cn) or Xiaochun Bai (baixc15@smu.edu.cn)

These authors contributed equally: Chuangxin Lin, Liangliang Liu, Chun Zeng
}

Received: 11 April 2018 Revised: 2 November 2018 Accepted: 14 November 2018

Published online: 20 February 2019 
mTORC2. mTORC1 is a sensitive target of rapamycin and is suppressed by the functional complex, tuberous sclerosis complex $1 / 2($ TSC $1 / 2) .{ }^{20}$ Accumulating evidences suggest that mTORC 1 is essential for maintenance of chondrocyte metabolic homeostasis and its activation in articular chondrocyte plays a vital role in OA development. ${ }^{21}$ We have previously shown that mTORC1 coordinates chondrocyte growth, proliferation, and differentiation, and that mTORC1 activation in turn activates articular cartilage chondrocytes and initiates $O A$, while its deletion or inhibition in chondrocyte prevents OA development. ${ }^{22,23}$ Previous studies by our group and others have also shown that balanced mTORC1 activity is critical for bone metabolism and development, acting by regulating osteoblast and osteoclast proliferation, differentiation, and function. ${ }^{24-26}$ However, the role of mTORC1 in subchondral bone formation during the pathogenesis of $\mathrm{OA}$ and the underlying mechanisms have not been reported.

$\mathrm{C}-\mathrm{X}-\mathrm{C}$ motif chemokine $12(\mathrm{Cxcl} 12)$, also known as the stromal cell-derived factor 1 (SDF1), is an 8-kDa chemokine originally isolated from bone marrow stromal cells. ${ }^{27}$ It regulates a wide variety of cellular activities via interactions with $\mathrm{C}-\mathrm{X}-\mathrm{C}$ chemokine receptor type 4 (CXCR4). ${ }^{28}$ Recently, increasing evidences indicate that Cxcl12/CXCR4 may play a role in the progression of OA. A dramatic increase of $\mathrm{CxCl} 12$ is found in the synovial fluid in the knee joints of rheumatoid arthritis and OA patients. ${ }^{29}$ Secondly, in vitro experiments have demonstrated that $\mathrm{CxCl} 12$ regulates chondrocyte catabolic activity by stimulating the release of matrix metalloproteinase-3 (MMP-3) and MMP-13. ${ }^{29,30}$ These findings strongly suggest that $\mathrm{CxCl} 12$ induces articular cartilage matrix degeneration during $\mathrm{OA}$ development. However, the mechanism by which $\mathrm{Cxcl} 12$ is regulated in OA remains to be elucidated.

In this study, we demonstrate that mTORC1 is activated in the preosteoblasts of subchondral bone from $\mathrm{OA}$ patients and destabilized OA mice. mTORC1 activation in Osterix ${ }^{+}$cells induces preosteoblasts to form aberrant clusters resulting in osteogenesis in subchondral bone, while inhibition of $\mathrm{MTORC} 1$ attenuates these pathological changes and reduces the degeneration of articular cartilage. mTORC1 activation of subchondral preosteoblasts promotes $\mathrm{OA}$ by stimulating aberrant subchondral bone formation and secretion of $\mathrm{Cxcl} 12$ to promote cartilage degeneration; thus, pharmaceutical inhibition of the pathway might be a promising therapeutic approach for OA treatment.

\section{RESULTS}

Preosteoblasts accumulate in subchondral bone during $O A$ development

To investigate the role of subchondral bone changes in $O A$, articular cartilage and subchondral bone specimens from $O A$ patients undergoing total knee replacement surgery or from nonOA trauma patients were analyzed. Hematoxylin and eosin (H\&E) and Safranin O-Fast green staining revealed aberrant bone formation and severe bone sclerosis in the tibial subchondral bone of OA patients (Supplementary Fig. 1a-c), which were confirmed by micro-computed tomography three-dimensional (3D) reconstruction and analysis (Supplementary Fig. $1 \mathrm{~h}$ and i). Tibial subchondral bone volume/total volume (BV/TV) fraction was positively correlated with $\mathrm{OA}$ Research Society International (OARSI) scores $(r=0.863, P<0.001)$ (Supplementary Fig. 1j). Importantly, the number of Osterix $^{+}$cells was dramatically increased in OA patients compared with that of non-OA control (Supplementary Fig. 1d-e), while the number of subchondral osteoclasts remained unchanged (Supplementary Fig. 1f-g), indicating that uncoupled subchondral bone modeling occurred during OA progression.

To further evaluate subchondral bone changes during $O A$ progression, the anterior cruciate ligament $(A C L)$ of mice was transected to generate a destabilized ACL-transected (ACLT) OA mouse model. Proteoglycan loss and articular cartilage degeneration were detected at 2 weeks after ACLT and progressed gradually (Fig. 1a-c). Interestingly, enhanced subchondral bone resorption in the tibial subchondral bone was observed at 2 weeks after ACLT, but aberrant bone formation and severe sclerosis occurred at the late stages of OA (Fig. 1a), as manifested by an increase in osteoclast number as well as larger subchondral bone marrow cavities at 2 weeks, and subsequently a decrease in the osteoclast number to baseline at 6 weeks after surgery (Fig. 1d, e). The number of Osterix ${ }^{+}$cells, however, was continuously elevated from 2 to 12 weeks after surgery (Fig. 1f, g), which may result from enhanced proliferation of Osterix ${ }^{+}$cells (Fig. 1h, i). Immunofluorescent analysis of Ki-67 in cultured primary preosteoblasts and mouse articular cartilage was used to rule out the false-positive results (Supplementary Fig. 2a). Micro-CT scan and analysis further confirmed that the BV/TV of tibial subchondral bone had decreased at 2 weeks, but was increased both at 6 and 12 weeks post surgery (Fig. 1j, k). Similarly, bone mineral density (BMD) was decreased at 2 weeks, but increased at 12 weeks post surgery (Fig. 1l). These findings further indicate that subchondral bone preosteoblasts are activated during $\mathrm{OA}$, resulting in aberrant bone formation and sclerosis.

mTORC1 is activated in subchondral bone preosteoblasts from OA patients and destabilized OA mice

Recent studies have shown that expression of mTOR is increased in peripheral blood mononuclear cells of OA patients, and is related to disease activity, ${ }^{31}$ and that pharmacological inhibition or genetic deletion of mTOR in chondrocyte decreases the severity of OA in an animal model. ${ }^{16,32}$ However, whether or not mTORC1 is activated in subchondral bone, and its role in OA has not been reported. Western blot analysis of subchondral bone tissues from collected tibial plateau specimens showed an enhanced phosphorylation of $\mathrm{S} 6(\mathrm{~S} 235 / 236)$ ( $\mathrm{p}-\mathrm{S} 6$, a target and marker of mTORC1 activation) in OA patients compared to that of control (Fig. 2a). Consistently, immunohistochemical staining confirmed that the number of $\mathrm{p}$-S6-positive cells dramatically increased in the subchondral bone of OA patients (Fig. 2b, d).

Activation of mTORC1 was also detected in tibial subchondral bone tissues isolated from ACLT mice ${ }^{33}$ at 2,6 , and 12 weeks post surgery (Fig. 2a). P-S6 staining revealed a dramatic increase of $\mathrm{p}$ S6-positive cells as early as 2 weeks post surgery (Fig. $2 b$, e). Moreover, most p-S6-positive cells were located on the subchondral bone surface in both OA patients and mice. Osterix and $\mathrm{p}-\mathrm{S} 6$ double staining confirmed that $\mathrm{p}-\mathrm{S} 6$ was detected mainly in Osterix ${ }^{+}$preosteoblasts in the subchondral bone of OA patients and $\mathrm{OA}$ mice (Fig. 2c, f, g). In addition, mTORC1 activation could also be detected in some mature osteoblasts $\left(\mathrm{OCN}^{+}\right)$during $\mathrm{OA}$ development in ACLT mice (Supplementary Fig. 2b-c). Taken together, these results indicate that mTORC1 is activated in subchondral bone, specifically in osteoblastic lineage cells in $\mathrm{OA}$ patients and mice.

Constitutive activation of $\mathrm{mTORC} 1$ in preosteoblasts induces aberrant bone formation in subchondral bone and promotes OA in mice

To characterize the specific role of subchondral bone mTORC1 activation in OA development, we generated mice with inducible mTORC1 activation in preosteoblast cells committed to the osteoblast lineage. We crossed floxed TSC1 (mTORC1-negative regulator) mice with Osx1-GFP::Cre mice (which express a GFP-Cre fusion protein under the direction of the Osx promoter and doxycycline) to generate conditional TSC1-knockout mice. Male mice with the genotype Osx1-GFP::CreTG $/{ }^{+} ; \mathrm{TSC}^{\text {flox/flox }}(\Delta \mathrm{TSC} 1)$ were selected for further studies. Male $\mathrm{TSC}^{\text {flox/flox }}$ littermates served as control (Supplementary Fig. 3a-b). To induce Osx-Credirected TSC1 disruption, doxycycline was omitted from the drinking water of mice at 12 weeks of age. The ablation of TSC1 


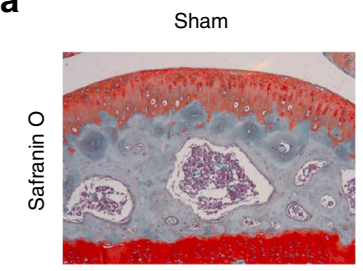

b

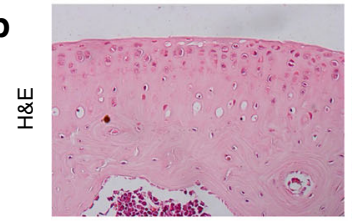

d

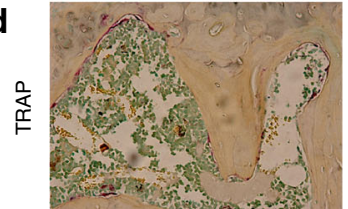

f
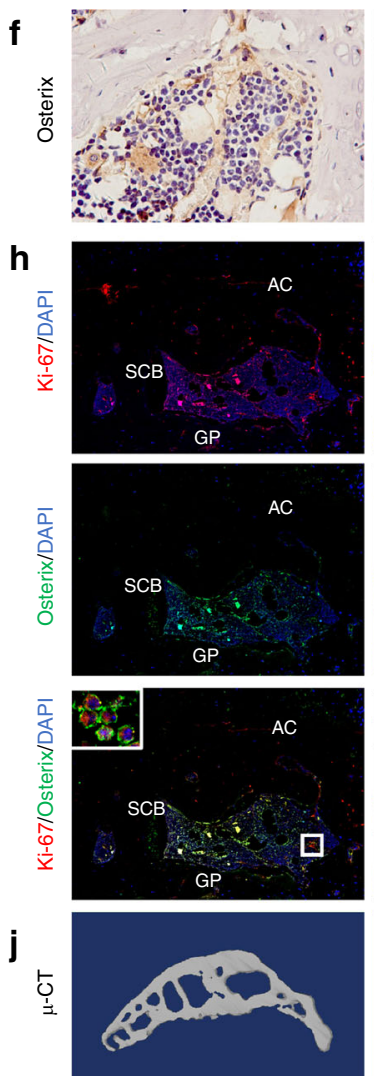

2 weeks
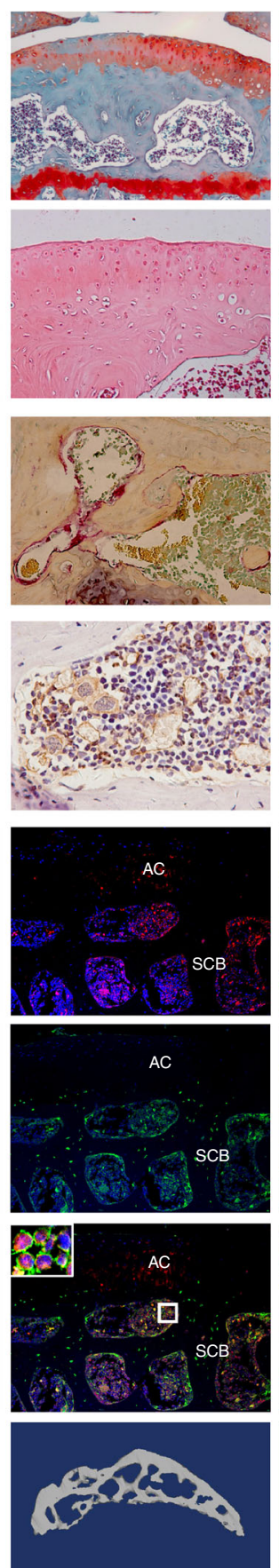

6 weeks
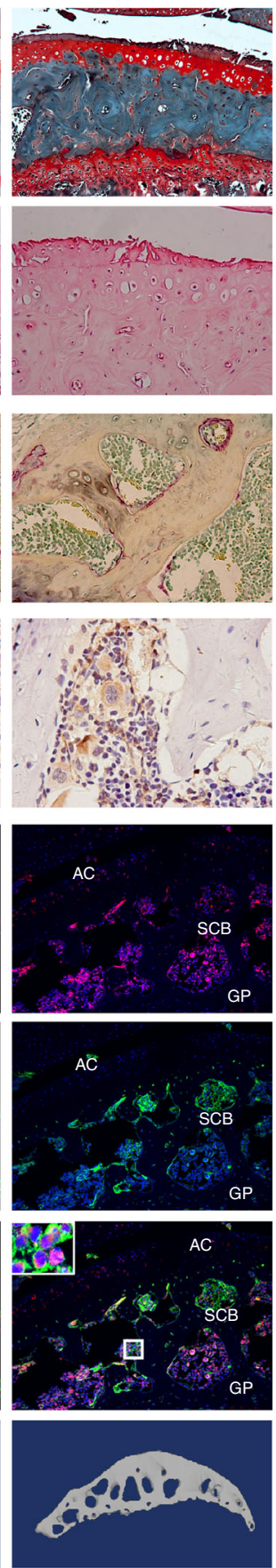

12 weeks
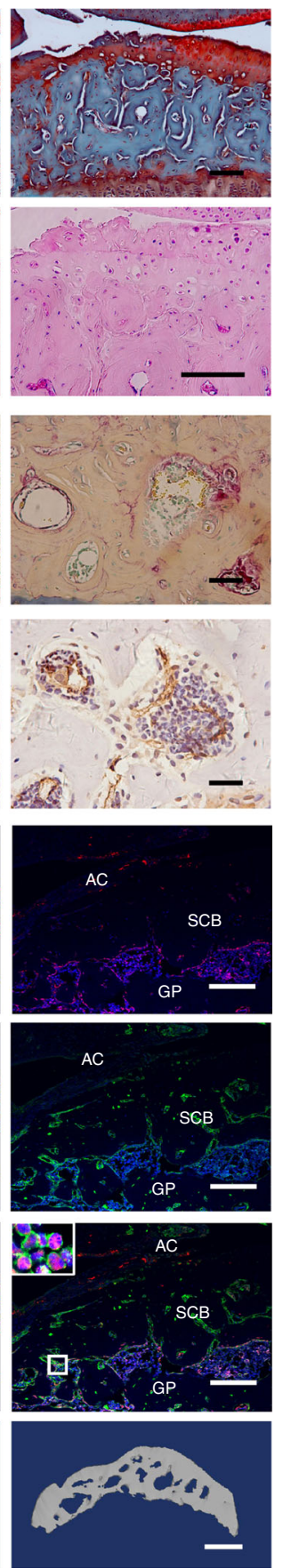

C
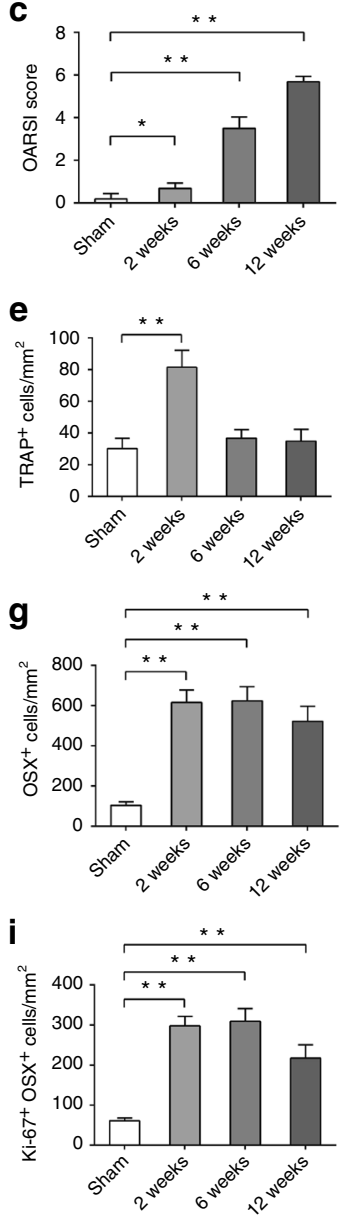

k
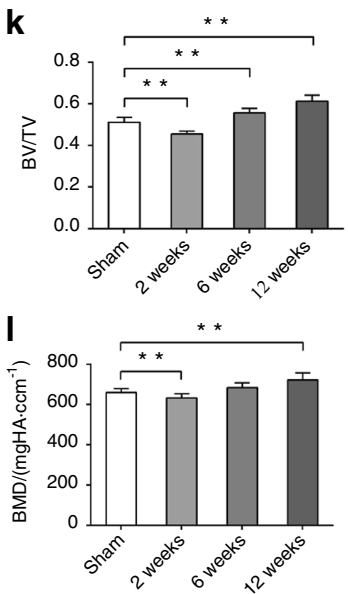

Fig. 1 Preosteoblasts accumulate and activate in subchondral bone of ACLT OA mice. a, -b Representative Safranin O-Fast green and H\&E staining of sagittal sections of tibia articular cartilage and subchondral bone in ACLT OA mice. Scale bars, $100 \mu \mathrm{m}$. c OARSI scores at 2, 6 , and 12 weeks post surgery compared with that of the sham control. d, e TRAP staining and quantitative analysis of osteoclasts in mouse tibial subchondral bone at 2,6, and 12 weeks post ACLT surgery compared with that of the sham control. Scale bars, $50 \mu \mathrm{m}$. $\mathbf{f}, \mathbf{g}$ Representative immunohistochemical images and quantitative analysis of Osterix ${ }^{+}$preosteoblasts in mouse tibial subchondral bone at 2,6 , and 12 weeks post ACLT surgery compared with that of the sham control. Scale bars, $100 \mu \mathrm{m}$. $\mathbf{h}, \mathbf{i}$ Immunostaining images and quantitative analysis of Ki-67 in Osterix ${ }^{+}$preosteoblasts in mouse tibial subchondral bone at 2,6 , and 12 weeks post ACLT surgery compared with that of the sham control. Boxed area is magnified on the top left corner. Scale bars, $100 \mu \mathrm{m}$. AC, articular cartilage; SCB, subchondral bone; GP, growth plate. $\mathbf{j}$ Representative 3D reconstructed micro-CT images of sagittal sections of mouse tibia subchondral bone at 2, 6, and 12 weeks post ACLT surgery compared with that of the sham control. Scale bars, $1 \mathrm{~mm}$. $\mathbf{k}$, I Quantitative analysis of bone mass in subchondral bone: bone volume/ total volume (BV/TV) and bone mineral density (BMD). Data are shown as mean \pm s.d. and analyzed by one-way ANOVA with Bonferroni post hoc test. $n=8,{ }^{* * P}<0.01$ 

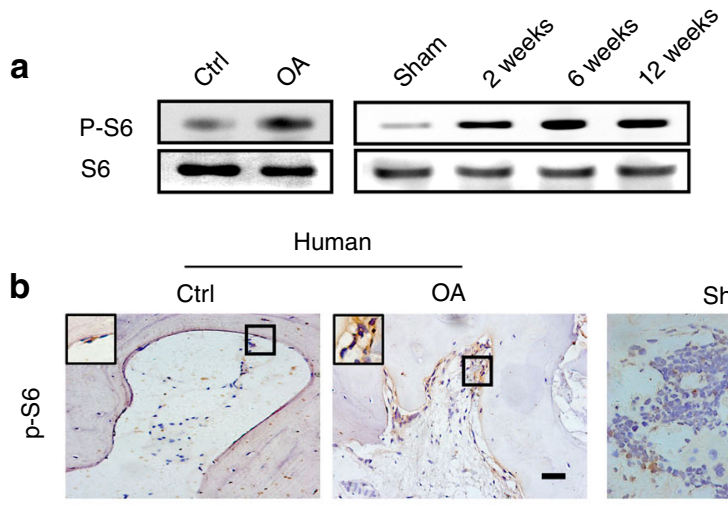

uman
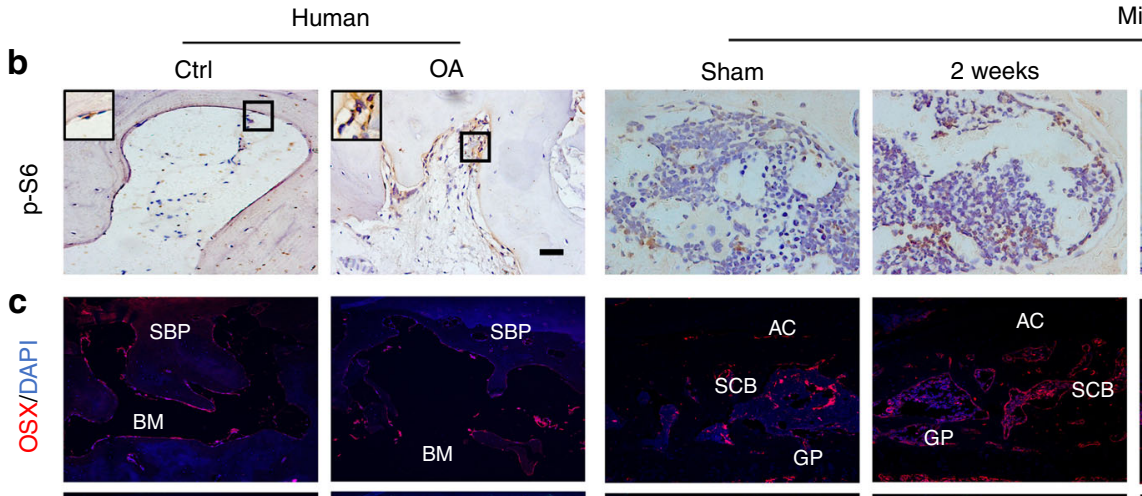

Mice
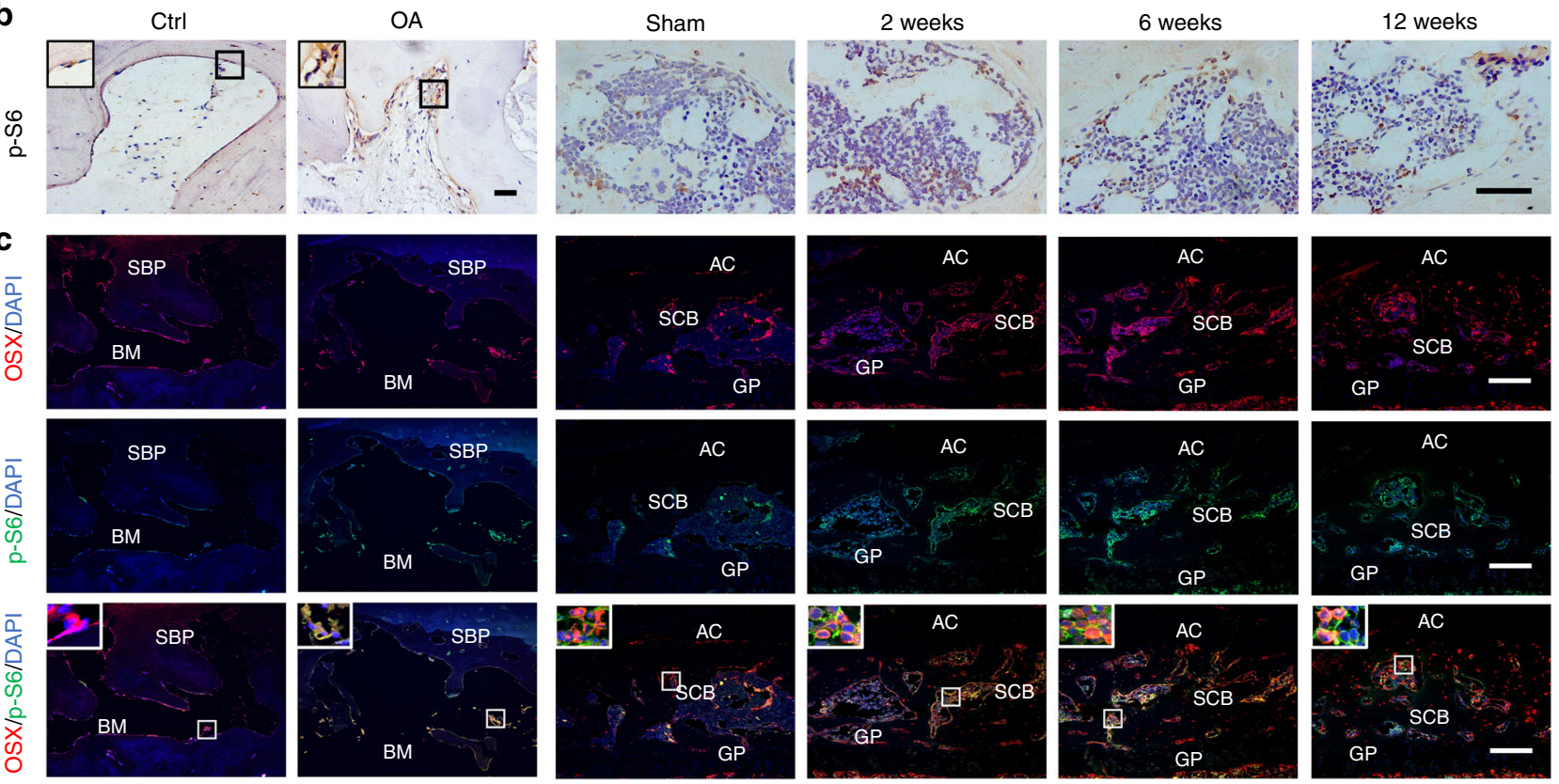

d

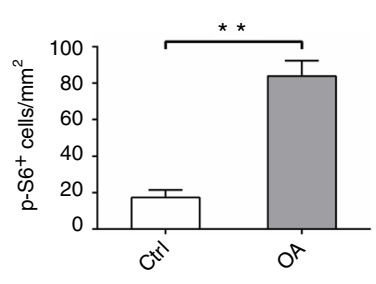

e

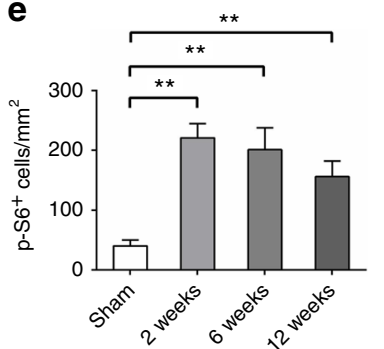

f

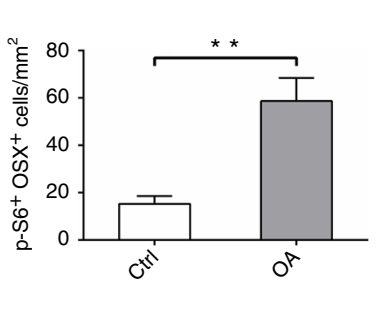

g

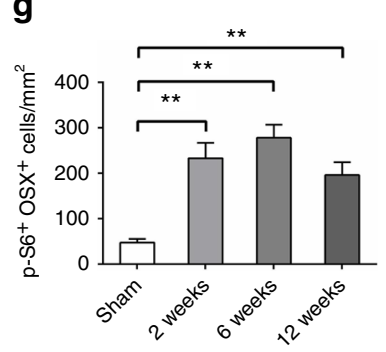

Fig. 2 mTORC1 is activated in subchondral bone preosteoblasts in OA patients and destabilized OA mice. a Western blot analysis of p-S6 expression in tibia subchondral bone tissues from patients and OA mice. $\mathbf{b}, \mathbf{d}, \mathbf{e}$ ) Representative immunostaining and quantitative analysis of $\mathrm{p}-\mathrm{S} 6^{+}$cells in tibial subchondral bone of OA patients and OA mice. Scale bars, $200 \mu \mathrm{m}$. c, f, g Representative immunostaining and quantitative analysis of p-S6 in Osterix ${ }^{+}$preosteoblasts in tibial subchondral bone of OA patients and OA mice. Boxed area is magnified on the top left corner. Scale bars, $100 \mu \mathrm{m}$. SBP, subchondral bone plate; BM, bone marrow; AC, articular cartilage; SCB, subchondral bone; GP, growth plate. Data are shown as mean \pm s.d. and analyze by Student's $t$ test or one-way ANOVA . $n \geq 8,{ }^{* *} P<0.01$

was validated by western blot analysis of tibial subchondral bone tissue (Supplementary Fig. 3c). Osterix and p-S6 double staining of tibial subchondral bone showed a dramatic increase in $\mathrm{p}$-S6 level in preosteoblast cells in $\triangle \mathrm{TSC} 1$ mice (Fig. 3a, b), indicating that mTORC1 was activated by TSC1 disruption.

Six weeks post TSC1 deletion, the subchondral bone BV/TV and $\mathrm{BMD}$ in $\triangle \mathrm{TSC} 1$ mice were both remarkably increased relative to that of their control (Supplementary Fig. 3d-f). The number of Osterix $^{+}$cells and proliferative Osterix ${ }^{+}$cells in subchondral bone were also dramatically increased, forming cell clusters on trabeculae surface (Supplementary Fig. $3 g-h$ and Fig. 3c, d). In contrast, the number of tartrate-resistant acid phosphatase (TRAP)-positive osteoclasts remained unchanged (Supplementary Fig. $3 g, i)$. Notably, significant aberrant bone formation and sclerosis could be observed in subchondral bone in sham $\triangle \mathrm{TSC} 1$ mice at 6 weeks post deletion (Fig. 3e).
To further validate the role of mTORC1 activation of Osterix ${ }^{+}$ cells in OA development, mature male $\triangle \mathrm{TSC} 1$ mice were subjected to ACLT and the knee joints were harvested 6 weeks post surgery. As excepted, $\triangle \mathrm{TSC} 1$ mice developed severe sclerosis of the subchondral bone, with almost all of the marrow cavities filled with aberrant newly formed bone (Fig. 3e), and both BV/TV and BMD were increased in ACLT $\triangle \mathrm{TSC} 1$ mice compared to that of the littermates or sham $\triangle \mathrm{TSC} 1$ mice (Fig. $3 \mathrm{f}-\mathrm{h}$ ). Notably, articular cartilage degeneration was aggravated in $\triangle \mathrm{TSC} 1$ mice following ACLT compared with that of the littermates or sham $\triangle T S C 1$ mice (Fig. 3e, i). Taken together, in the absence of surgery, $\triangle \mathrm{TSC} 1$ mice presented subchondral bone sclerosis with little-to-no effects on articular cartilage integrity. On the other hand, $\triangle \mathrm{TSC} 1$ mice receiving ACLT surgery exhibited more severe cartilage damage compared to that in the control and sham-operated $\triangle \mathrm{TSC} 1$ mice. Thus, subchondral bone sclerosis driven by deletion of TSC1 in 

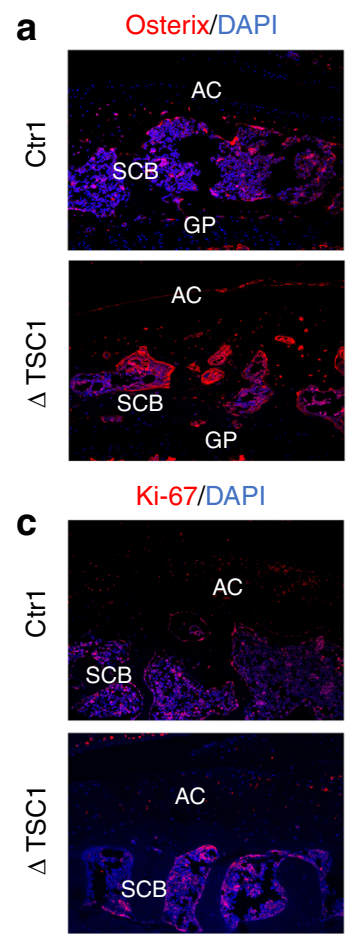

e
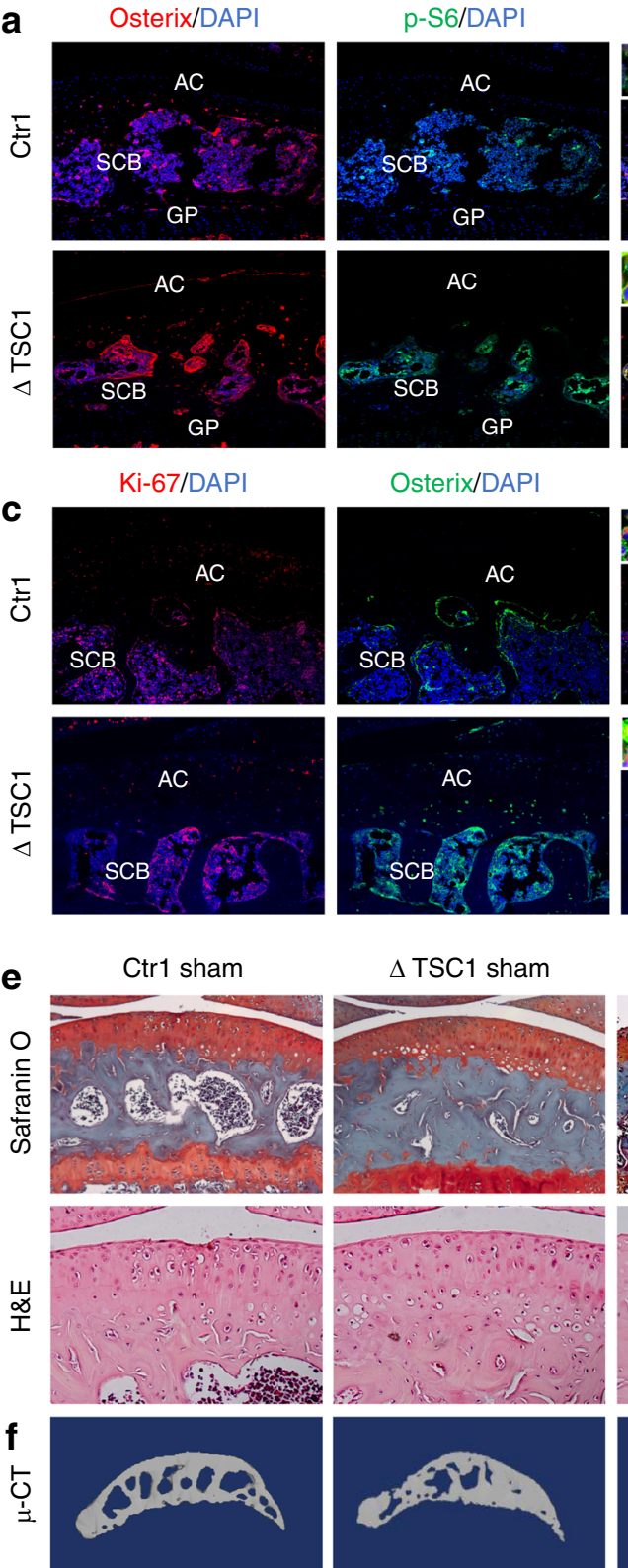

Osterix/DAPI
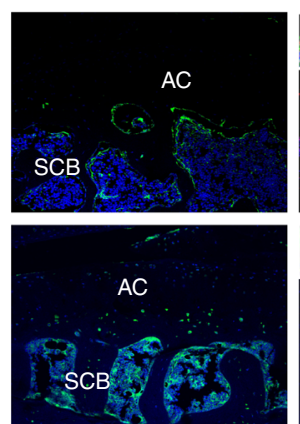

$\triangle$ TSC1 sham
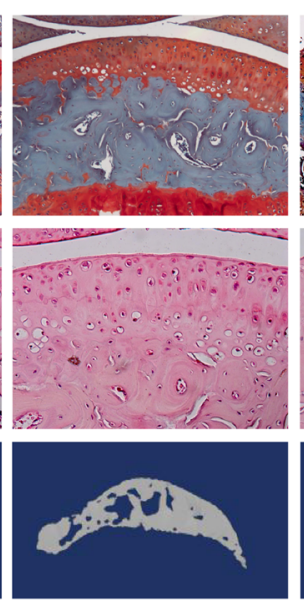

Osterix/p-S6/DAPI
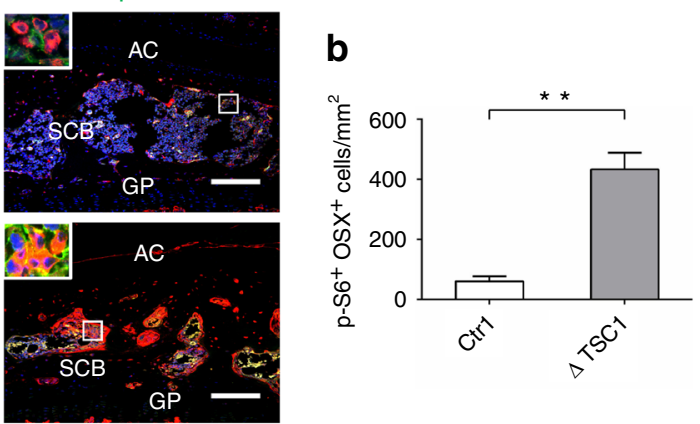

Ki-67/Osterix/DAPI

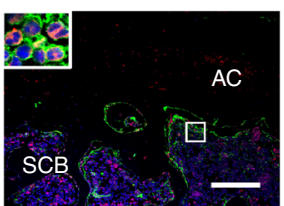

\title{
d
}
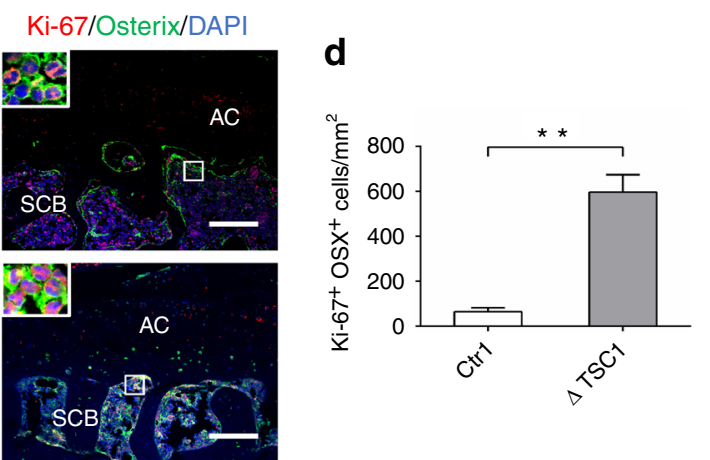

\begin{abstract}
Ctr1 ACLT
\end{abstract}

\section{$\triangle \mathrm{TSC} 1 \mathrm{ACLT}$}
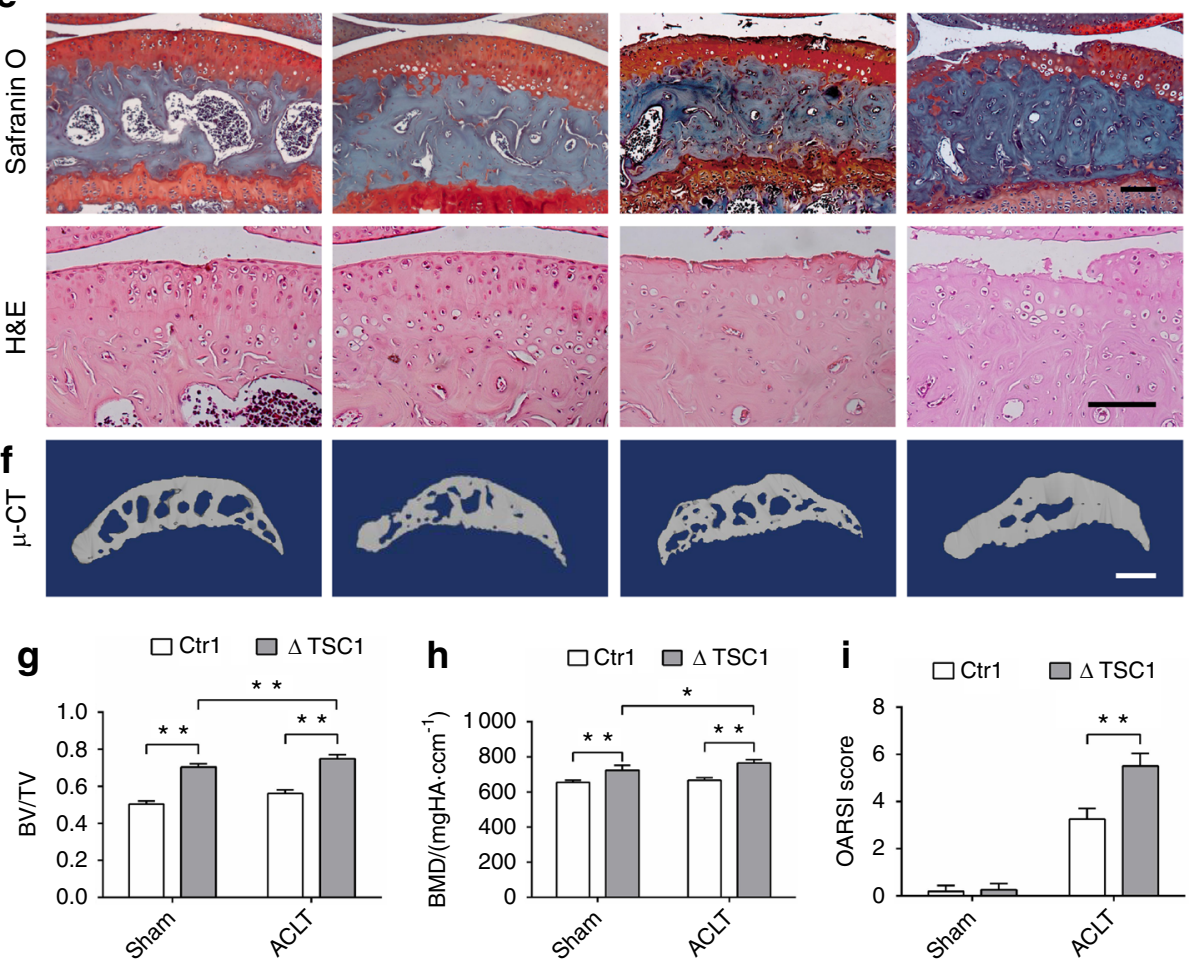

Fig. 3 Constitutive activation of $\mathrm{mTORC} 1$ in preosteoblasts induces aberrant bone formation in subchondral bone and promotes OA in mice. a, b Representative photomicrographs and quantification of immunostaining of $\mathrm{p}-\mathrm{S} 6$ in Osterix ${ }^{+}$preosteoblasts in tibial subchondral bone of transgenic mice after Tsc1 deletion ( $\triangle \mathrm{TSC} 1$ ) compared to that of their littermates (Ctrl). c, d Representative photomicrographs and quantification of immunostaining of $\mathrm{Ki}-67$ in Osterix ${ }^{+}$preosteoblasts in tibial subchondral bone of transgenic mice after Tsc1 deletion ( $\Delta$ TSC1) compared to that of their littermates (Ctrl). Boxed area is magnified on the top corner. Scale bars, 100 $\mu \mathrm{m}$. AC, articular cartilage; SCB, subchondral bone; GP, growth plate. e Representative Safranin O-Fast green and H\&E staining of sagittal sections of articular cartilage and tibia subchondral bone of $\triangle \mathrm{TSC} 1$ sham mice and $\triangle \mathrm{TSC} 1$ ACLT mice compared to that of their littermates (Ctrl). Scale bars, $100 \mu \mathrm{m}$. f Representative 3D reconstructed micro-CT images of sagittal of tibia subchondral bone of $\Delta$ TSC1 sham mice and $\Delta$ TSC1 ACLT mice compared to that of their littermates (Ctrl). Scale bars, $1 \mathrm{~mm} . \mathbf{g}, \mathbf{h}$ Quantitative analysis of bone mass in subchondral bone: bone volume/total volume (BV/TV) and bone mineral density (BMD). i OARSI scores based on the histology analysis. Data are shown as mean \pm s.d. and analyzed by Student's $t$ test or two-way ANOVA . $n \geq 6,{ }^{*} P<0.05$, and ${ }^{* *} P<0.01$ 
preosteoblasts is not sufficient to induce $O A$, but can accelerate disease progression following surgical destabilization of the joint.

Deletion of mTORC1 in preosteoblasts prevents aberrant subchondral bone formation and OA development

To further investigate the role of subchondral bone preosteoblast mTORC1 activation in OA development, we generated mice with ablation of Raptor (a key mTORC1 component) in preosteoblast cells. We crossed floxed Raptor mice with Osx1-GFP::Cre mice to generate Osx1-GFP::CreTG $/{ }^{+}$; Raptor ${ }^{\text {flox/flox }}$ ( $\Delta$ Raptor) mice (Supplementary Fig. 4a-b). Doxycycline was omitted from the drinking water of mature $\Delta$ Raptor mice to delete Raptor in Osterix ${ }^{+}$cells. The inactivation of mTORC1 was further confirmed by western blot and Osterix and p-S6 double staining (Supplementary Fig. 4c and Fig. 4a, b), indicating that mTORC1 was inhibited by Raptor deletion.

In contrast to mice with mTORC1 activation in preosteoblast cells, trabecular bone loss and large bone marrow cavities were observed in the subchondral bone of $\Delta$ Raptor mice with a 6-week inducible deletion (Supplementary Fig. 4d), and the BV/TV and BMD were significantly reduced (Supplementary Fig. $4 \mathrm{e}-\mathrm{f}$ ). The number of Osterix $^{+}$cells and proliferative Osterix ${ }^{+}$cells in the subchondral bone marrow of $\Delta$ Raptor mice was significantly less than that of control (Supplementary Fig. $4 \mathrm{~g}-\mathrm{h}$ and Fig. 4c, d), with no change in the osteoclast number (Supplementary Fig. $4 \mathrm{~g}$ and i). Notably, articular cartilage proteoglycan remained intact in the $\Delta$ Raptor mice post sham surgery (Fig. 4e).

To validate whether mice with mTORC1 inhibition in preosteoblasts could prevent subchondral aberrant bone formation and OA development, $\triangle$ Raptor mice were similarly subjected to ACLT and the knee joints were harvested at 6 weeks post surgery. Interestingly, the microarchitecture of subchondral bone was stabilized, as the BV/TV and BMD were significantly decreased in ACLT $\triangle$ Raptor mice compared with that of the littermates, but not significantly different compared with that of the sham $\Delta$ Raptor mice (Fig. 4f-h), indicating that aberrant subchondral bone formation was prevented during OA progression. Furthermore, surgically induced degeneration of articular cartilage was also attenuated relative to control mice receiving ACLT surgery (Fig. 4e, i). Taken together, these results further consolidated the theory that mTORC 1 activation in Osterix ${ }^{+}$cells plays a critical role in aberrant subchondral bone formation and sclerosis, and suggest that targeted inhibition of mTORC1 in subchondral Osterix $^{+}$cells could prevent cartilage degeneration and posttraumatic OA development.

\section{Activation of mTORC1 in subchondral preosteoblasts produces} Cxcl12, promoting cartilage degradation

To explore the mechanisms through which subchondral mTORC1 in preosteoblasts promotes $\mathrm{OA}$, we created a global messenger RNA (mRNA) expression profile of TSC1-defected or control preosteoblasts using a microarray. Among the up-regulated mRNAs, Cxcl12 has been reported to play a role in cartilage degeneration. We found that $\mathrm{CxCl} 12$ was expressed constitutively in preosteoblast cells in vitro (Fig. 5a). We further confirmed the increase ( $>20$-folds) of $\mathrm{Cxcl} 12$ mRNA in primary $\triangle \mathrm{TSC} 1$ preosteoblasts, but found a decrease ( $>5$-folds) of Cxcl12 mRNA in $\triangle$ Raptor cells versus control cells (Fig. $5 \mathrm{a}$ ). Cxcl12 protein levels exhibited similar changes in cultured preosteoblasts. Moreover, activation of mTORC1 promoted $\mathrm{CxCl} 12$ expression and inactivation of mTORC1 reduced $\mathrm{Cxcl}_{12}$ expression in $\mathrm{Osterix}^{+}$preosteoblasts both in subchondral bone and primary spongiosa in metaphysis (Fig. 6a, b, d, e). Furthermore, the secretion of $\mathrm{Cxcl} 12$ in culture supernatant of primary $\triangle \mathrm{TSC} 1$ preosteoblasts was up-regulated, while its secretion in $\Delta$ Raptor cells was down-regulated compared with that of control cells (Fig. 5b). Interestingly, serum Cxcl12 level was significantly increased in wild-type mice post ACLT surgery (Fig. 5c), and further increased in ACLT $\triangle T S C 1$ mice, but significantly decreased in ACLT $\triangle$ Raptor mice (Fig. 5d). Altogether, these findings demonstrate that $\mathrm{mTORC} 1$ positively regulates Cxcl12 expression and secretion in preosteoblasts, implying that changes in $\mathrm{Cxcl} 12$ level caused by mTORC1-expressing preosteoblasts may contribute to the phenotypes of articular cartilage degeneration in $\triangle \mathrm{TSC} 1$ and $\triangle \mathrm{TSC} 1 \mathrm{ACLT}$ mice described above.

We next sought to determine whether $\mathrm{Cxcl} 12$ was responsible for articular cartilage degeneration during OA. Interestingly, CXCR4, a CxCl12 receptor, was constitutively expressed in articular cartilage chondrocytes of $\triangle \mathrm{TSC} 1, \triangle$ Raptor mice, and their respective control (Fig. 5e). We further treated ACLT $\triangle T S C 1$ mice with anti-Cxcl12 antibody to neutralize endogenous $\mathrm{CxCl} 12$ in vivo. Notably, articular cartilage degeneration was significantly attenuated by administration of the antibody compared to those treated with normal immunoglobulin G (IgG) (Fig. 6c, f). Moreover, the percentages of MMP- $13^{+}$and type $\mathrm{X}$ collagen ${ }^{+}$chondrocytes were significantly reduced (Fig. $6 \mathrm{c}, \mathrm{g}, \mathrm{h}$ ), indicating protection from cartilage degeneration. Consistently, ADTC5 cells maintained in conditioned medium (CM) from $\triangle \mathrm{TSC} 1$ preosteoblasts exhibited hypertrophic differentiation and mineralization, compared with those cultured in $\mathrm{CM}$ from $\Delta$ Raptor and control preosteoblasts (Fig. 5f, h). The hypertrophic differentiation of ATDC5 cells induced by CM from TSC1-deficient preosteoblasts was significantly attenuated by $\mathrm{Cxcl} 12$-neutralizing antibody (Fig. $5 \mathrm{~g}$, i). These data suggest that the elevated $\mathrm{Cxcl} 12$ in preosteoblasts contributes to articular cartilage degeneration in TSC1-deficient ACLT mice.

Interestingly, CXCR4 was also detected in mesenchymal stem cells (MSCs) (Fig. 5e), and MSCs maintained in CM from $\triangle \mathrm{TSC} 1$ preosteoblasts or supplemented with $\mathrm{Cxcl} 12$ exhibited osteogenic differentiation and mineralization, compared with those cultured in CM from control preosteoblasts (Fig. 5j). The effect of TSC1-deficient preosteoblasts CM on ATDC5 cells was significantly attenuated by $\mathrm{Cxcl} 12$-neutralizing antibody (Fig. 5j). Moreover, alleviative bone formation and bone sclerosis were observed in the subchondral bone by administration of anti-Cxcl12 antibody (Fig. 6C), as well as evidenced by decreased $\mathrm{OCN}^{+}$cells, BV/TV, and BMD compared to those treated by normal mouse IgG (Fig. 6i-k). These data suggested that Cxcl12 may contribute to aberrant subchondral bone remodeling during OA development, in addition to articular cartilage degeneration.

\section{DISCUSSION}

Although the contribution of abnormal subchondral bone remodeling to articular cartilage degeneration and OA progression has been established, ${ }^{34}$ the precise mechanism by which aberrant subchondral bone formation is initiated and induces cartilage degeneration during $O A$ is still unclear. Previously, it was shown that subchondral bone-derived tumor growth factor- $\beta$ and hypoxia-inducible factor1a could be released, causing uncoupling of subchondral bone remodeling and damage to articular cartilage in destabilized OA mouse models. ${ }^{1,35,36}$ These studies demonstrate that subchondral bone changes may be more sensitive to alterations in mechanical loading, and reveal the contribution of subchondral bone changes to articular cartilage degeneration in OA progression; however, the cellular and molecular mechanisms of such communication are not well defined. In the present study, we show the critical role of mTORC1 signaling by preosteoblasts in subchondral bone formation, and cross-talk to articular cartilage degeneration mediated by Cxcl12 during post-traumatic OA development. Inactivation of mTORC1 in subchondral bone preosteoblasts or treatment with Cxcl12 antibody is able to prevent subchondral bone sclerosis and attenuate articular cartilage degeneration in OA mice. Therefore, this study, the first report on mTORC1 signaling in aberrant subchondral bone formation and the interplay between subchondral bone and articular cartilage during $\mathrm{OA}$ progression, shows that targeted inhibition of preosteoblast $\mathrm{mTORC} 1$ provides a novel prospective therapy for OA. 
a
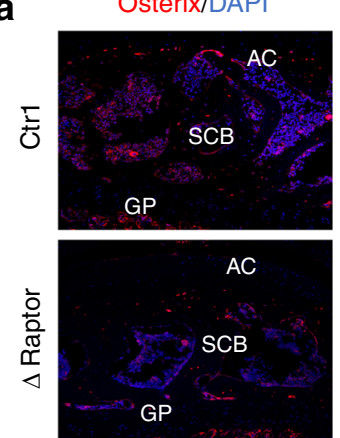

Ki-67/DAP

C
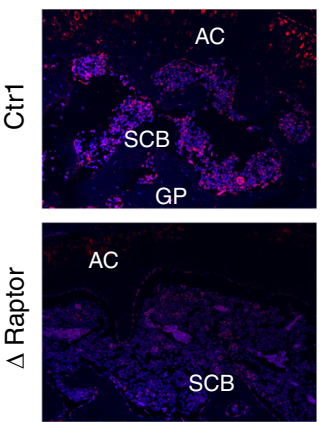

e

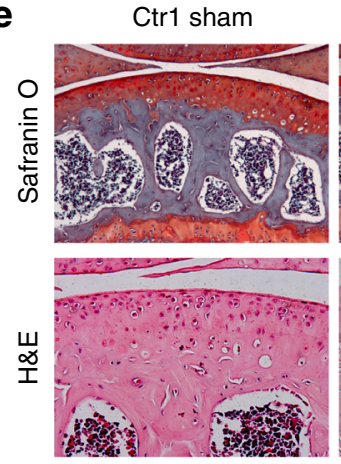

$\mathbf{f}$

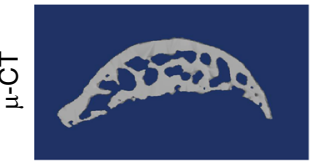

p-S6/DAPI

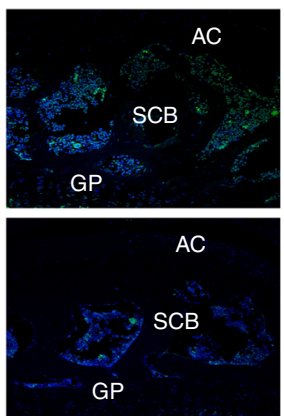

Osterix/DAPI
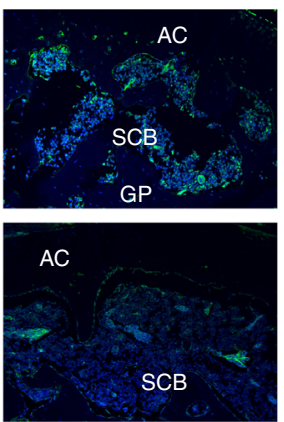

$\Delta$ Raptor sham
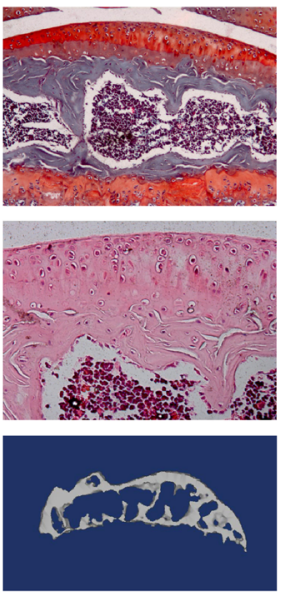

Osterix/p-S6/DAPI

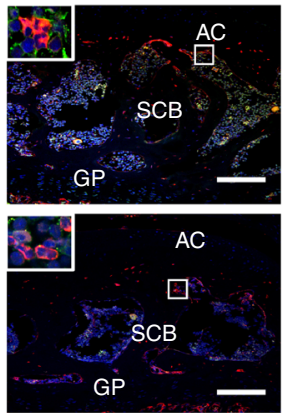

Ki-67/Osterix/DAPI
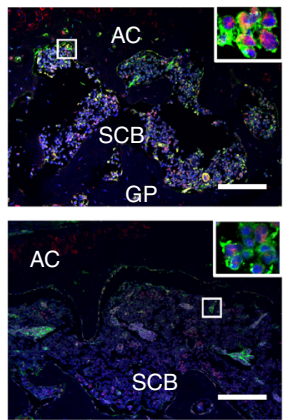

Ctr1 ACLT
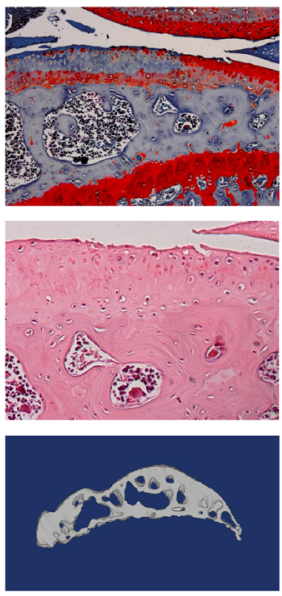

b

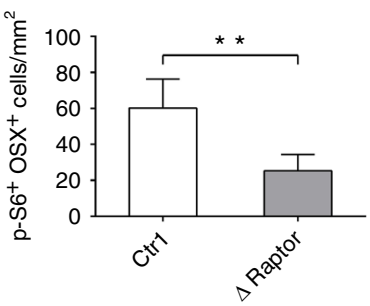

d

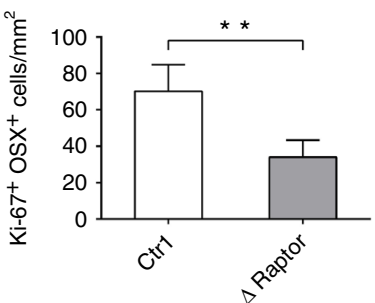

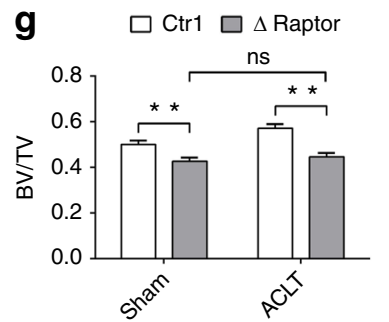
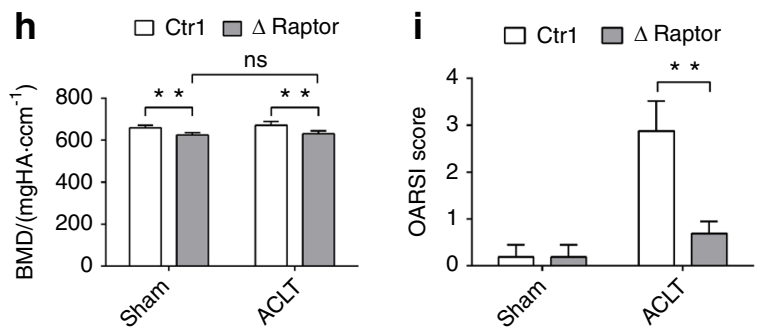

Fig. 4 Inhibiting mTORC1 signaling in preosteoblasts prevents aberrant subchondral bone formation and OA development. a, b Representative photomicrographs and quantification of immunostaining of $\mathrm{p}-\mathrm{S} 6$ in Osterix ${ }^{+}$preosteoblasts in tibial subchondral bone of transgenic mice after Raptor deletion ( $\Delta$ Raptor) compared to that of their littermates (Ctrl). c, d Representative photomicrographs and quantification of immunostaining of Ki-67 in Osterix ${ }^{+}$preosteoblasts in tibial subchondral bone of transgenic mice after Raptor deletion ( $\Delta$ Raptor) compared to that of their littermates (Ctrl). Boxed area is magnified on the top corner. Scale bars, $100 \mu \mathrm{m}$. AC, articular cartilage; SCB, subchondral bone; GP, growth plate. e Representative Safranin O-Fast green and H\&E staining of sagittal sections of articular cartilage and tibia subchondral bone of $\Delta$ Raptor sham mice and $\Delta$ Raptor ACLT mice compared to that of their littermates (Ctrl). Scale bars, $100 \mu$ m. f Representative 3D reconstructed micro-CT images of sagittal sections of tibia subchondral bone of $\Delta$ Raptor sham mice and $\Delta$ Raptor ACLT mice compared to that of their littermates (Ctrl). Scale bars, $1 \mathrm{~mm} . \mathbf{g}$, $\mathbf{h}$ Quantitative analysis of bone mass in subchondral bone: bone volume/ total volume (BV/TV) and bone mineral density (BMD). i OARSI scores based on the histology analysis. Data are shown as mean \pm s.d. and analyzed by Student's $t$ test or two-way ANOVA. $n \geq 6,{ }^{* *} P<0.01$, and $n s \geq 0.05$ 

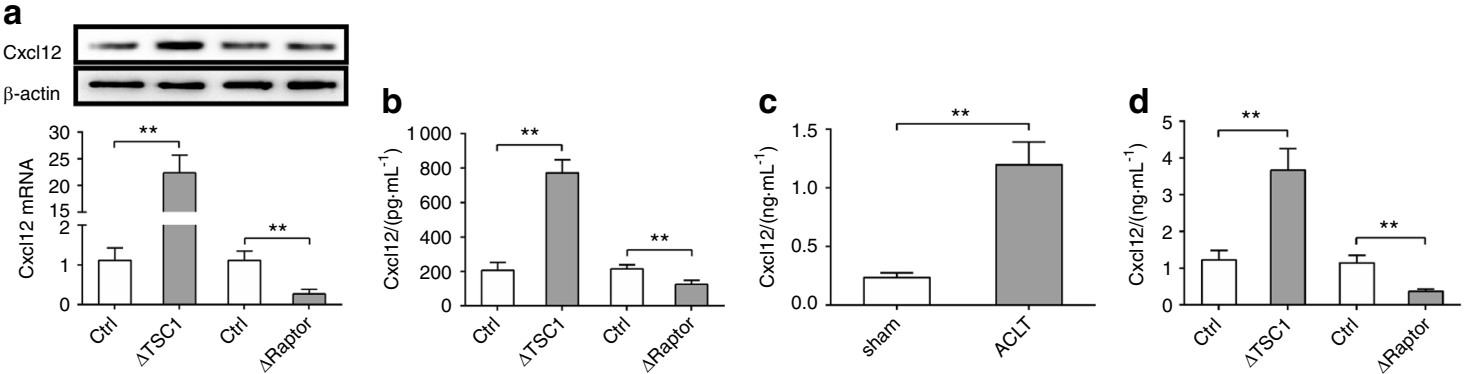

e

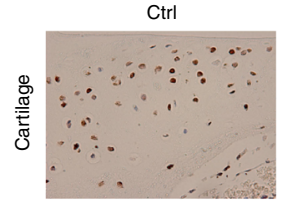

$\Delta \mathrm{TSC} 1$
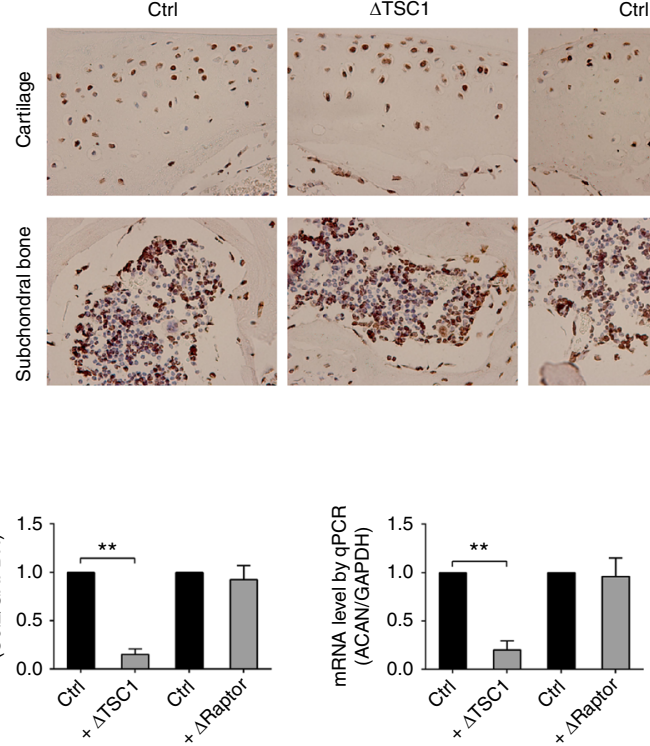

Ctrl
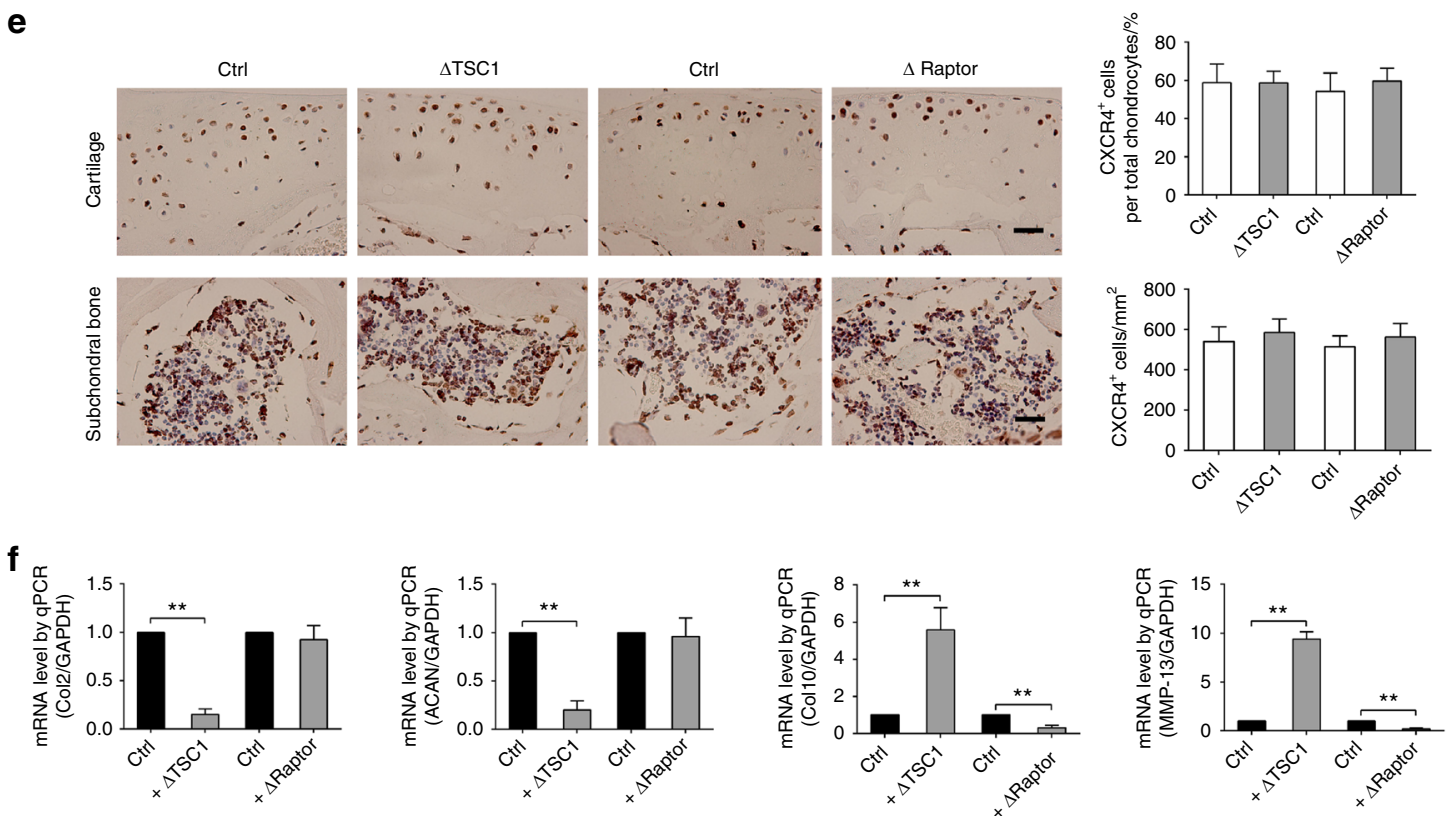

g
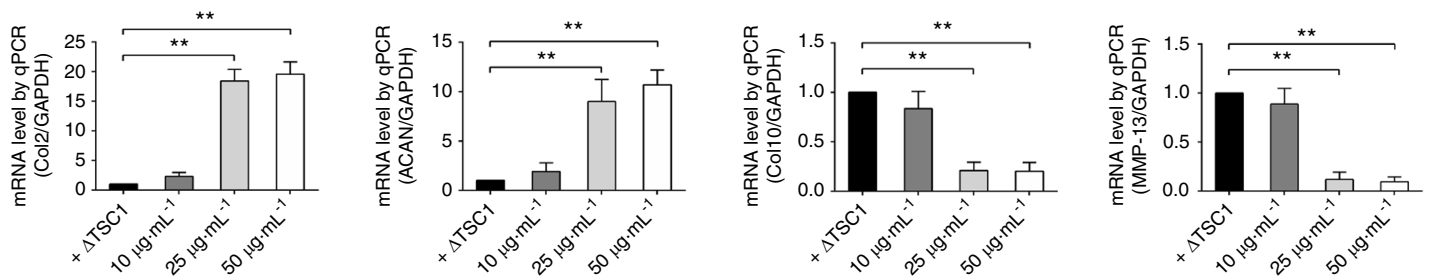

n

i

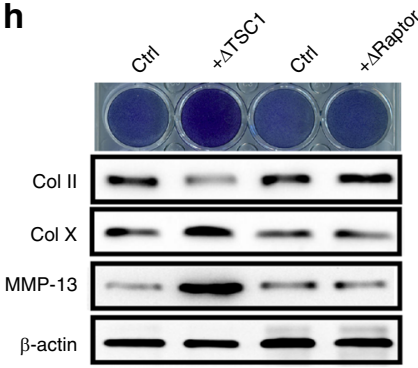

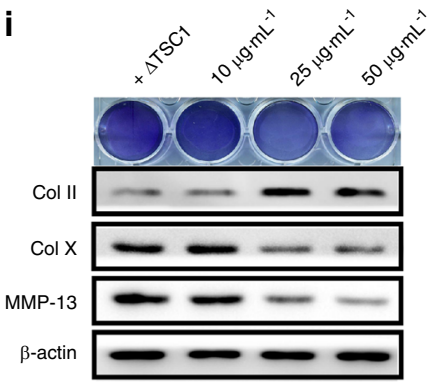

j

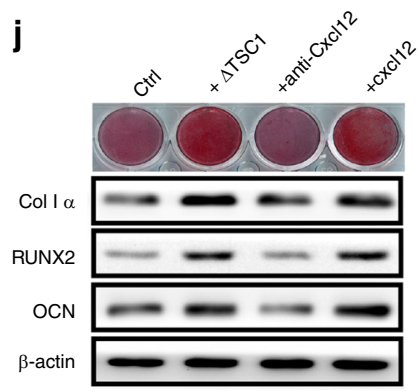

Fig. 5 mTORC1 activation in subchondral preosteoblasts produce $\mathrm{Cxcl} 12$ to promote chondrocyte hypertrophic differentiation MSCs osteogenic differentiation. a Western blot and quantitative PCR analysis of Cxcl12 in primary preosteoblasts. b-d) Cxcl12 assessed by ELISA in the cultured medium of primary preosteoblasts or in the serum at 6 weeks post ACLT surgery. e Representative immunostaining and quantitative analysis of CXCR4 ${ }^{+}$cells in articular cartilage and subchondral bone in $\triangle T S C 1, \Delta$ Raptor mice, and their littermates (Ctrl). Scale bars, $100 \mu \mathrm{m}$. Quantitative analysis of CXCR4 ${ }^{+}$cells out of total cartilage chondrocytes. $\mathbf{f}$, $\mathbf{g}$ Quantitative PCR analysis of Col2, ACAN, Col X, MMP-13 mRNA in ADTC5 cells treated with the cultured medium of primary preosteoblasts and Cxcl12-neutralizing antibody for 7 days. $\mathbf{h}, \mathbf{i}$ Western blot analysis of Col2, Col X, MMP-13, and Toluidine blue staining in ADTC5 cells treated with CM of primary preosteoblasts and Cxcl12-neutralizing antibody for 7 days. $\mathbf{j}$ Western blot analysis of Col $1 \alpha$, Col X, MMP-13, and Alizarin red staining in ADTC5 cells treated with $\mathrm{CM}$ of primary preosteoblasts, recombinant murine $\mathrm{CxCl} 12$ or $\mathrm{CxCl} 12$-neutralizing antibody for 14 days. Data are shown as mean \pm s.d. and analyzed by Student's $t$ test and one-way ANOVA . $n \geq 8,{ }^{* *} P<0.01$ 

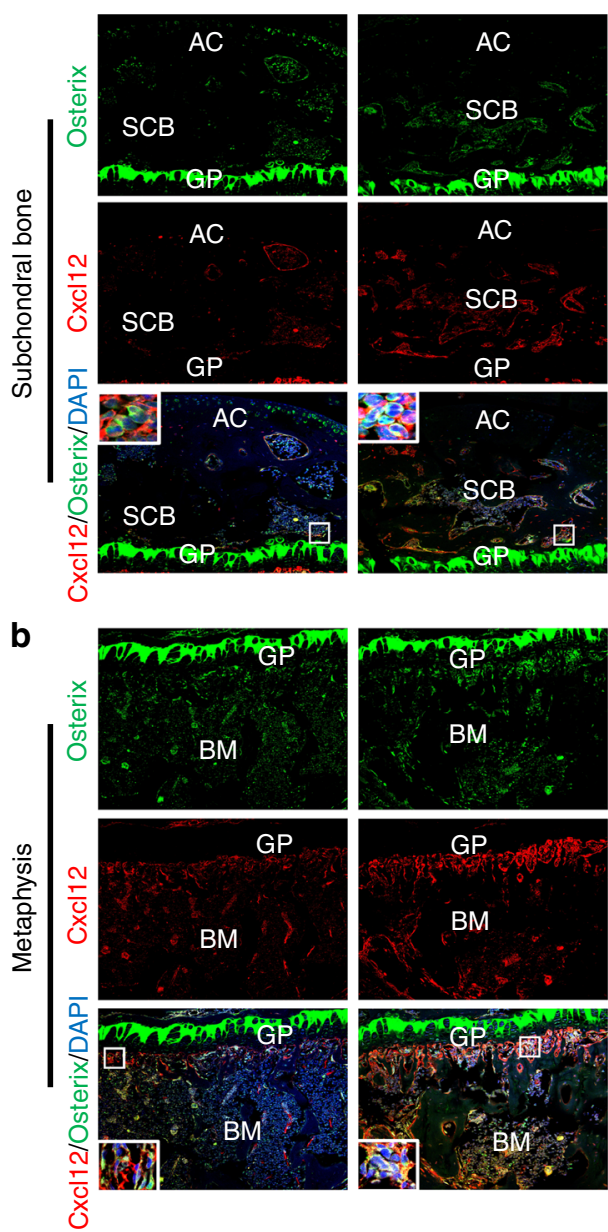

d

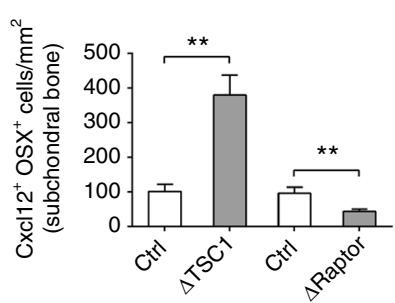

h

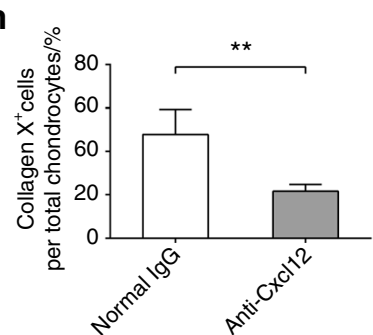

$\Delta \mathrm{TSC} 1$
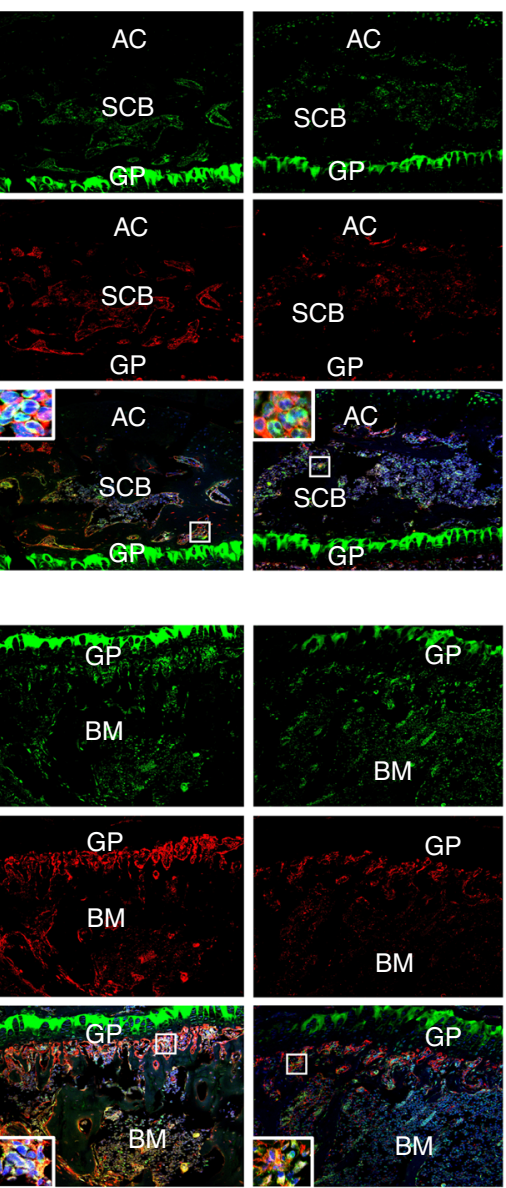

e

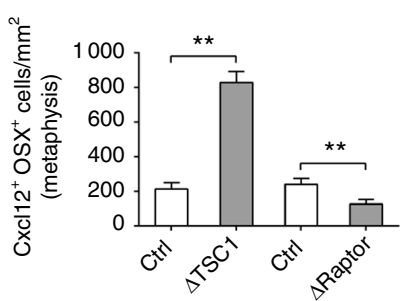

i

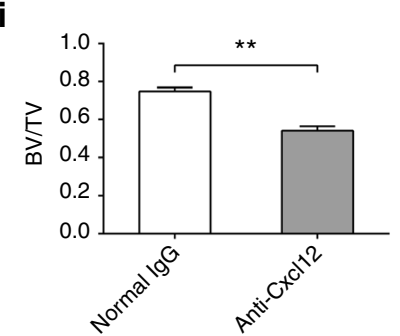

$\Delta$ Raptor
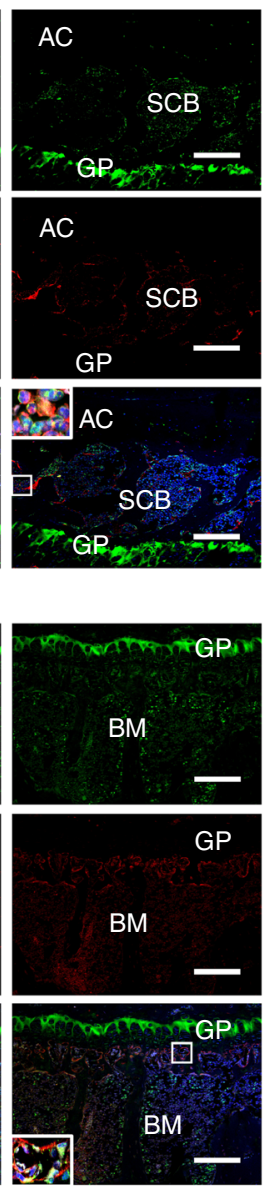

f
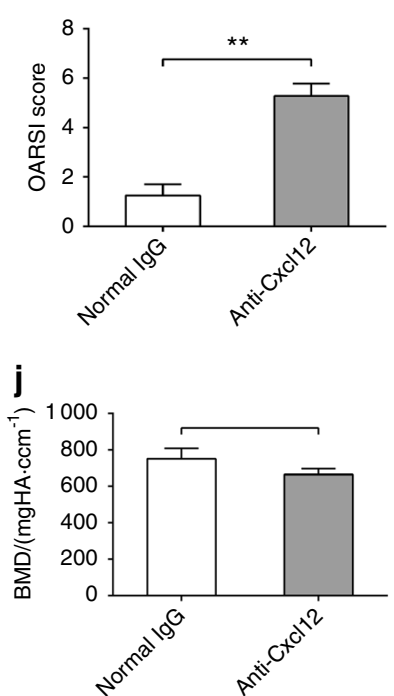
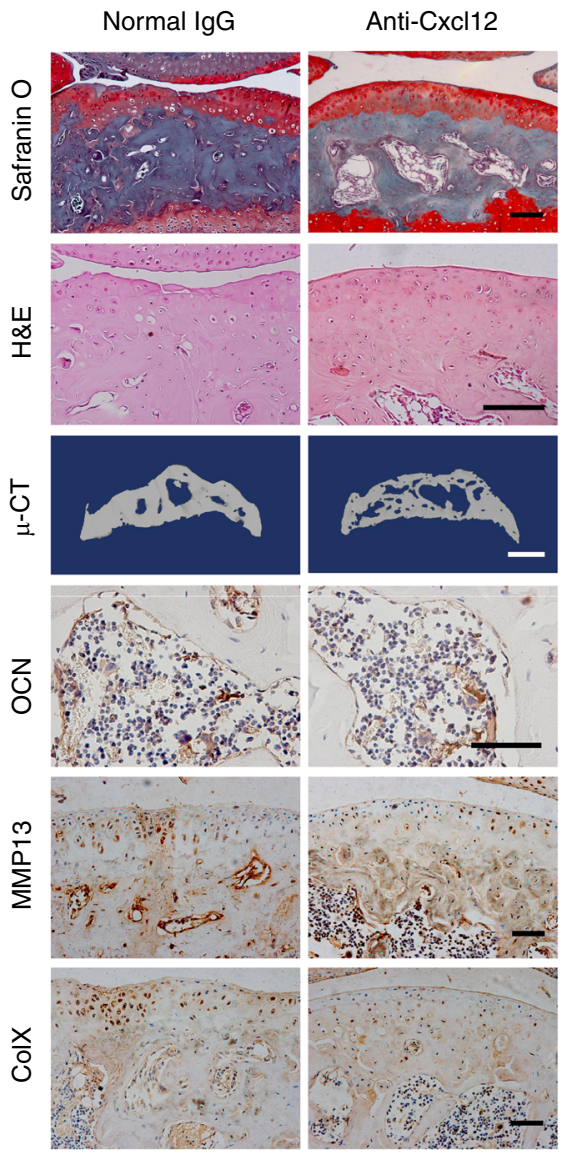

Fig. 6 Administration of anti-Cxcl12 antibody attenuated subchondral bone remodeling and articular cartilage degeneration in ACLT mice. a, b, d, e Representative immunostaining and quantitative analysis of $\mathrm{Cxcl} 12$ in Osterix ${ }^{+}$preosteoblasts in the subchondral bone and metaphysis in $\triangle T S C 1, \Delta$ Raptor mice, and their littermates (Ctrl). Boxed area is magnified on the corner. Scale bar, $100 \mu \mathrm{m}$. AC, articular cartilage; SCB, subchondral bone; GP, growth plate; BM, bone marrow. c Representative Safranin O-Fast green, H\&E staining, 3D reconstructed $\mu$-CT images and immunohistochemical staining of OCN, type $X$ collagen, and MMP-13 in $\triangle T S C 1$ mice administered with anti-Cxcl12 antibody or normal IgG for 6 weeks. Scale bar, $100 \mu \mathrm{m}$ (Safranin O, H\&E staining, OCN, type X collagen, and MMP-13), 1 mm ( $\mu$-CT). f OARSI scores based on the histological analysis. $\mathbf{g}, \mathbf{h}$ Quantitative analysis of type $\mathbf{X}$ collagen and MMP-13 in articular cartilage. $\mathbf{i}$, $\mathbf{j}$ Quantitative analysis of bone mass in subchondral bone: bone volume/total volume (BV/TV) and bone mineral density (BMD). $\mathbf{k}$ Quantitative analysis of OCN ${ }^{+}$osteoblasts in tibial subchondral bone. Data are shown as mean \pm s.d. and analyzed by Student's $t$ test. $n \geq 6,{ }^{* *} P<0.01$ 
Both clinical and animal studies have reported that progression of $\mathrm{OA}$ is accompanied by turnover in the subchondral bone microarchitecture. ${ }^{10,37}$ Aberrant subchondral bone formation and sclerosis have been reported in human OA joints ${ }^{38}$ and in several experimental OA models. ${ }^{39}$ Similarly, we found that the subchondral bone underwent dramatic remodeling after surgery in ACLT mice and OA patients. The identified risk factors for OA, including ligament injury, excessive body weight, and muscle weakening during aging, increase the mechanical loading on weight-bearing joints. ${ }^{40-42}$ The architecture of subchondral bone and plate adapt via modeling and remodeling in response to these mechanical stresses. Because the subchondral bone acts as a structural girder and shock absorber for the adjacent articular cartilage, aberrant bone formation and sclerosis enhance the subchondral bone holding strength and stiffness, but reduce elastic deformation and shock absorption capacity, causing an imbalance in mechanical stress distribution and/or excessive loading in articular cartilage, which in turn causes articular cartilage wear as a result of joint motion. ${ }^{35,43}$ Preventing aberrant bone formation and sclerosis in subchondral bone is a promising therapeutic strategy to curb the progression of OA.

mTORC1 is known for its anabolic effects on articular cartilage homeostasis by stimulating the production of extracellular matrix proteins and preventing terminal differentiation of chondrocytes, ${ }^{23}$ but the role of mTORC1 in subchondral bone changes during $\mathrm{OA}$ is unclear. In this study, we observed that changes in mechanical loading increased mTORC1 activity in subchondral bone preosteoblasts in mice and in vitro. In addition, mTORC1 is also activated in subchondral bone preosteoblasts from OA patients. As we and other groups have shown that mTORC1 activation in Osterix $^{+}$cells strongly promotes preosteoblast proliferation and expansion and produces high bone mass in mice, ${ }^{25,44}$ mTORC1 activation may stimulate subchondral preosteoblast proliferation and sustained subchondral bone formation in ACLT and $\triangle T S C 1$ mice. Notably, during the normal remodeling process, osteoblasts and their progenitors are located at resorption sites on the bone surface. However, abnormal mechanical loading-induced mTORC1 activation of subchondral preosteoblasts promotes their proliferation and "in situ" formation of preosteoblast clusters in the bone marrow cavities. We suggest that these clustered preosteoblasts, spatiotemporally uncoupled from osteoclastic bone resorption, produce large amount of less mineralized bone in the bone marrow, leading to subchondral bone sclerosis. These data reveal that mTORC1 plays an essential role in aberrant subchondral bone formation and sclerosis during OA development.

Previous studies have shown that $\mathrm{Cxcl} 12$ is a catabolic factor that can infiltrate cartilage, reduce proteoglycan content, and increase MMP-13 activity. ${ }^{45}$ Inhibiting the CXCl12/CXCR4 signaling pathway can attenuate the pathogenesis of OA. ${ }^{46}$ Moreover, it also induces the migration of bone marrow MSCs to articular lesion sites. For the first time, we established the existence of cross-talk between bone-forming preosteoblasts and articular chondrocytes mediated by $\mathrm{CxCl} 12$. Cxcl12 was expressed constitutively in preosteoblasts and regulated by mTORC1. Furthermore, serum $\mathrm{Cxcl} 12$ level was drastically up-regulated by mTORC1 activation of preosteoblasts during $\mathrm{OA}$, which was detected in ACLT mice. Its receptor CXCR4 was expressed in articular cartilage chondrocytes. We speculated that a direct effect of $\mathrm{Cxcl} 12$ on these cells might contribute to articular cartilage degeneration observed in our mouse models. Indeed, chondrocytes and preosteoblasts cocultured in vitro generated similar effects to those observed in articular cartilage when exposed directly to $\mathrm{Cxcl} 12$ in vivo. Importantly, Cxcl12-neutralizing antibody administration reduced cartilage degeneration and alleviated OA development in OA and mutant mice. These results support our conclusion that $\mathrm{CxCl} 12$ mediates articular cartilage degeneration stimulated by mTORC1 activation in subchondral preosteoblasts during OA.
In order to investigate the role of mTORC1 activation in subchondral bone remodeling during post-traumatic OA development, the ACLT OA mouse model was employed in this study according to previous studies. ${ }^{1,47}$ However, ACLT model might be too severe to represent human OA. The DMM model is more reliable, reproducible, and structural similar to human degenerative OA. ${ }^{48}$ The mTORC1 activation in the DMM model is of interest for further investigation.

Taken together, the results of this study suggest a novel pathological mechanism for OA development on subchondral bone-articular cartilage interplay. Abnormal mechanical loading activates $\mathrm{mTORC} 1$ in subchondral bone preosteoblasts in $\mathrm{OA}$, and mTORC1 activation promotes preosteoblast proliferation and Cxcl12 secretion, resulting in aberrant subchondral bone formation and bone sclerosis, and articular cartilage degeneration (Fig. 7). Consequently, pharmaceutical inhibition of the pathways might be beneficial for OA patients.

\section{MATERIALS AND METHODS}

Human samples

After the study was approved by the Ethics Committee of the Third Affiliated Hospital of Southern Medical University, 38 tibial plateaus (including articular cartilage) were obtained from OA patients who were undergoing knee replacement surgery. Eight normal samples from victims of road traffic accidents with no history of arthritic diseases served as control. The specimens were processed for micro-CT analysis, histological examination, and calculated per patient in three sequential sections with OARSI scoring system as previously described. ${ }^{49}$ The number of positively stained cells in the tibia subchondral bone area was counted in three sequential sections per patient in each group.

\section{Mice}

We purchased the Tsc1 $1^{\text {flox/flox }}$, Raptor flox/flox, R26-mT/mG, and Osx1-GFP::Cre mouse strains from the Jackson Laboratory (Bar Harbor, ME, USA). The background of Tsc1 $1^{\text {flox/flox }}$ mice is 129S4/ SvJae, and these mice were backcrossed to mice with a C57BL/6 background for eight generations before use. We performed genotyping using genomic DNA isolated from tail biopsies, using the following primers: loxP Tsc1 allele forward, 5'-GTCACGACC GTAGGAGAAGC-3' and reverse, 5'-GAATCAACCCCACAGAGCAT-3'; loxP Raptor allele forward, 5' -CTCAGTAGTGGTATGTGCTCAG-3' and reverse, 5'-GGGTACAGTATGTCAGCACAG-3'; Osx1-GFP::Cre forward, 5'-CTCTTCATGAGGAGGACCCT-3' and reverse, 5'GCCAGGCAGGTGCCTGGACAT-3'.

We purchased C57BL/6J (wild-type) mice from the Experimental Animal Centre of Southern Medical University (Guangzhou, China). Importation, transportation, housing, and breeding of the mice were all conducted according to the recommendations of "The use of non-human primates in research." The mice were euthanatized by cervical dislocation to prevent suffering. The Southern Medical University Animal Care and Use Committee approved all procedures involving the mice.

\section{Doxycycline treatment}

Osx-Cre (Osx1-GFP::Cre), a BAC transgenic mouse line expressing a GFP::Cre fusion protein from the regulatory sequence of Osx, was generated to direct gene deletion in the osteoblast lineage. ${ }^{50}$ The characterization of this mouse line revealed that Cre activity is largely restricted to the osteogenic perichondrium, periosteum, and osteoblast-lineage cells within the marrow cavity. ${ }^{51}$ Cremediated recombination in these mice is under the control of doxycycline. To prevent the Osx promoter from driving Cre expression, mated mice were fed with doxycycline (Sigma-Aldrich, St. Louis, MO, USA; 9891) in drinking water to deliver a daily dose of 2-3 mg/mouse of doxycycline according to the manufacturer's instruction. Cre activity was indeed suppressed in Osx-Cre mice by 


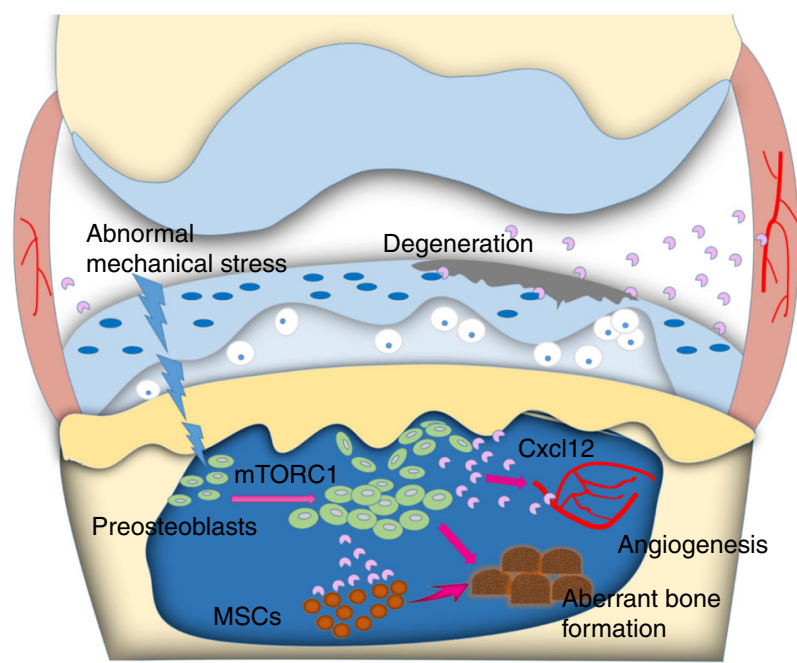

Fig. 7 Model of elevated active mTORC1 in the subchondral bone at the onset of OA. mTORC 1 is activated in the subchondral bone in response to abnormal mechanical loading, which induces preosteoblast abnormal proliferation and $\mathrm{Cxcl} 12$ secretion to promote subchondral bone formation and articular cartilage degeneration for OA progression

doxycycline treatment in Osx-Cre; R26-mT/mG mice (Supplementary Fig. 2d).

Experimental OA model, anti-Cxcl12 antibody treatment, and histomorphometry

Three-month-old male C57BL/6J, $\Delta \mathrm{TSC} 1, \Delta$ Raptor mice and control mice were subjected to $A C L$ transection (ACLT) surgical procedure to induce mechanical instability-associated $O A$, as previously described, ${ }^{52}$ and the procedure to establish sham operation group was similar to operation group but without transecting the ACL. Knee joints freshly dissected from mice were fixed for $48 \mathrm{~h}$ in $4 \%$ paraformaldehyde at $4{ }^{\circ} \mathrm{C}$, and then decalcified for 21 days in $10 \%$ EDTA ( $\mathrm{pH}$ 7.4). The tissues were embedded in paraffin and sectioned continuously ( $4 \mu \mathrm{m}$ thick) and serial sections were obtained from the medial and lateral compartments at $50-\mu \mathrm{m}$ intervals. Eight representative mid-sagittal sections were deparaffinized in xylene, hydrated with graded ethanol, and stained with H\&E or Safranin O/Fast Green. We quantified the degeneration of the articular cartilage of the medial and lateral tibial plateau joint with the OARSI scoring system as previously described. ${ }^{53}$ The final score was generated by averaging individual scores.

\section{Micro-CT analysis}

The knee joint specimens were analyzed by high resolution $\mu-C T$ Scanner (Viva CT80; Scanco Medical AG, Bassersdorf, Switzerland). The scanner was set at a voltage of $55 \mathrm{kV}$, a current of $145 \mathrm{~mA}$ and a resolution of $10 \mu \mathrm{m}$ per pixel. Cross-sectional images of the tibial subchondral bone were used to perform three-dimensional histomorphometric analysis. We defined the region of interest to cover the whole subchondral bone medial compartment, and we used a total of ten consecutive images from medial tibial plateau for 3D reconstruction and analysis. Three-dimensional structural parameters analyzed including: bone mineral density (BMD), BV/ TV, trabecular number and trabecular thickness.

Immunohistochemical and immunofluorescent analyses TRAP staining (Sigma-Aldrich, Missouri, USA) and immunostaining were performed according to the manufacturer's instructions. Serial sections were incubated with primary antibodies to antiOsterix (Abcam, ab22552; Santa Cruz, sc-393060), anti-Ki-67
(Immunoway, YM-3064), anti-p-S6 ribosomal protein (Ser235/ 236) (Cell Signaling Techonlogy, \#2211), anti-Cxcl12 (Abcam, ab9797), anti-CXCR4 (Abcam, ab124824), anti-collagen X (Abcam, ab58632), and anti-MMP-13 (Abcam, ab39012) overnight at $4{ }^{\circ} \mathrm{C}$. For immunofluorescent staining, secondary antibodies conjugated with fluorescent tags were added and slides were incubated at room temperature for $1 \mathrm{~h}$ in dark. Photomicrographs of sections were captured to perform histomorphometric measurements on the entire area of the tibial subchondral bone with ZEISS Scope A1 (Zeiss, Heidelberg, Germany), Quantitative histomorphometric analysis was conducted in a blinded fashion using the OsteoMeasure XP Software (OsteoMetrics, Inc., Atlanta, USA). The number of positively stained cells in the entire tibia subchondral bone area was counted in three sequential sections per mouse in each group.

\section{Cell culture}

The chondrogenic cell line ATDC5 (Riken BioResource Center, Tsukuba, Japan) was cultured in maintenance medium consisting of Dulbecco's modified Eagle's medium (DMEM) nutrient mixture F12 (DMEM:F12) (Gibco) supplemented with 5\% fetal bovine serum (FBS) (Gibco). Chondrogenesis of ATDC5 cells was induced by treatment with insulin, transferrin, and selenous acid (Sigma-Aldrich) according to a previously reported method. ${ }^{54}$ Bone marrow-mesenchymal stromal cells were isolated from bone marrow of $\mathrm{C} 57 \mathrm{BL} / 6$ mice and previously characterized by phenotyping and trilineage differentiation potential as described previously. ${ }^{55}$ They were expanded in proliferative medium consisting in DMEM, $100 \mu \mathrm{g} \cdot \mathrm{mL}^{-1}$ penicillin/streptomycin, $2 \mathrm{mmol} \cdot \mathrm{L}^{-1}$ glutamine, and supplemented with $10 \% \mathrm{FBS}$. For osteogenic induction, $50 \mu \mathrm{g} \cdot \mathrm{mL}^{-1}$ L-ascorbic acid (Sigma-Aldrich) and $10 \mathrm{mmol} \cdot \mathrm{L}^{-1} \beta$-glycerol phosphate (Sigma-Aldrich) were added.

Primary preosteoblast cells were isolated from the calvaria of newborn mice. In brief, calvariae were dissected from the mice (24h after birth), rinsed with phosphate-buffered saline (PBS) and digested in freshly prepared $0.1 \mathrm{mg} \cdot \mathrm{mL}^{-1}$ collagenase type-II (Thermo Fisher Scientific, Waltham, MA, USA) in a-minimal essential Eagle's medium (a-MEM) at $37^{\circ} \mathrm{C}$ for $20 \mathrm{~min}$; the digestion was repeated five times. After digestion, supernatants were combined and centrifuged to pellet the cells. ${ }^{56}$ Cells were then maintained in a-MEM (Gibco, Thermo Fisher Scientific) supplemented with $10 \%$ FBS (Gibco), $100 \mathrm{U} \cdot \mathrm{mL}^{-1}$ penicillin and $100 \mathrm{mg} \cdot \mathrm{mL}^{-1}$ streptomycin sulfate, at $37^{\circ} \mathrm{C}$ with $5 \% \mathrm{CO}_{2}$. After reaching confluence in $60-\mathrm{mm}$ culture dishes, the medium was replaced with a-MEM (Gibco) supplemented with $1 \%$ bovine serum albumin, and the cells were cultured for $16 \mathrm{~h}$ before collecting the CM. The CM was then centrifuged for $15 \mathrm{~min}$ at $1500 \times g$ at $4{ }^{\circ} \mathrm{C}$, and then stored at $-80^{\circ} \mathrm{C}$ until use. Mouse Cxcl12 antibody (R\&D Systems, Minneapolis, MN, USA, \#MAB310, 10-50 $\mu \mathrm{g} \cdot \mathrm{mL}^{-1}$ ) and recombinant murine Cxcl12 (PrimeGene BioTech, \#20315, $100 \mathrm{ng} \cdot \mathrm{mL}^{-1}$ ) was added to the $\mathrm{CM}$ as indicated.

Toluidine blue staining and Alizarin red staining Cultured cells were fixed with $4 \%$ paraformaldehyde for $30 \mathrm{~min}$ at room temperature, then stained using a Toluidine Blue Staining Kit (Leagene, Beijing, China) for $60 \mathrm{~min}$ at room temperature, and finally washed with PBS to remove excess dye.

For the detection of osteogenic differentiation, the Alizarin red assay (Sigma-Aldrich) was performed to determine mineralization. Briefly, cells were washed with PBS, fixed with paraformaldehyde for $30 \mathrm{~min}$, incubated with $1 \%$ Alizarin red for $30 \mathrm{~min}$ at room temperature, and washed with PBS to remove the excess of staining. Osteogenic nodules were stained in orange-red due to calcium deposition.

ELISA analysis

We used the mouse (SDF1) ELISA (enzyme-linked immunosorbent assay) Kit (Elabscience Biotechnology Co. Ltd, Wuhan, China; \#E- 
EL-M1094C) to analyze Cxcl12 in serum and CM. ELISA analysis was performed according to the manufacturer's instructions.

Real-time quantitative PCR and microarray analysis

Total RNA was isolated from cell pellets using TRIzol reagent (Life Technologies, Grand Island, NY, USA). Complementary DNA was reversely transcribed from RNA samples using reverse transcription reagents (Vazyme Biotech Co. Ltd, Nanjing, China) and quantitative PCR assays were carried out to quantify levels of mRNA expression of Cxcl12, type-II collagen (Col2), aggrecan (ACAN), type $X$ collagen (Col10), and collagenolytic MMP (MMP-13) with glyceraldehyde 3-phosphate dehydrogenase (GAPDH) as the internal loading control using Real-Time PCR Mix (Vazyme Biotech Co. Ltd) in a Light Cycler (Roche Molecular Biochemicals, Indianapolis, IN, USA). The following primer sequences were used: Cxcl12 (forward primer: 5'-TCCCCTTGTGTITGGCAGT-3'; reverse primer: 5'-TTGCATCTCCCACGGATGTC-3'); Col2 (forward primer: $5^{\prime}-$ CACACTGGTAAGTGGGGCAAGACCG-3'; reverse primer: $5^{\prime}$-GG ATTGTGTTGTTCAGGGTTCGGG-3'); ACAN (forward primer: $5^{\prime}-\mathrm{GA}$ AGGTGAAGGTCGGAGTC-3'; reverse primer: 5'-GAAGATGGTGAT GGGATTTC-3'); Col10 (forward primer: 5'-AAGTGGACCGAAAGGA GACA-3'; reverse primer: 5'-TGGAAACCCATTCTCACCTC-3'); MM P-13 (forward primer: 5'-GCTGCGGTTCACTTTGAGAA-3'; reverse primer, 5'-GGCGGGGATAATCTITGTCCA-3'); and GAPDH (forward primer: 5'-AAATGGTGAAGGTCGGTGTGAAC-3'; reverse primer, 5'CAACAATCTCCACTITGCCACTG-3').

For mRNA array analysis, samples were submitted to Shanghai Biotechnology Corporation for hybridization on an Agilent-014868 Whole Mouse Genome Microarray 4×44K G4122F (Probe Name version). Each microarray chip was hybridized to a single sample labeled with Cy3. Background subtraction and normalization were performed. Finally, mRNAs with expression levels differing by at least 3-folds between control and TSC1-defected preosteoblasts were selected $(P<0.05)$. Microarray data have been deposited in the GEO database under accession code GSE74781.

\section{Western blot analysis}

Cells were lysed with $2 \%$ sodium dodecyl sulfate (SDS), $2 \mathrm{~mol} \cdot \mathrm{L}^{-1}$ urea, $10 \%$ glycerol, $10 \mathrm{mmol} \cdot \mathrm{L}^{-1}$ Tris- $\mathrm{HCl}(\mathrm{pH} 6.8), 10 \mathrm{mmol} \cdot \mathrm{L}^{-1}$ dithiothreitol, and $1 \mathrm{mM}$ phenylmethylsulfonyl fluoride. The lysates were centrifuged and the supernatants were separated by SDS-polyacrylamide gel electrophoresis and blotted onto a nitrocellulose membrane (Bio-Rad Laboratories, Hercules, CA, USA). The membrane was then incubated with specific antibodies to phospho-S6 (S235/236) (Cell Signaling Techonlogy, \#2211), S6 (Santa Cruz Biotechnology Inc., sc-74459), phospho-AMPK (Thr172) (Cell Signaling Technology, \#2535), AMPK (Cell Signaling Technology, \#2532), Cxcl12 (Abcam, ab9797), collagen X (Abcam, ab58632), MMP-13 (Abcam, ab39012), collagen II (Abcam, ab185430), collagen I (Abcam, ab6308), osteocalcin (Santa Cruz Biotechnology Inc., sc-23790), RUNX2 (Abclonal, A2851), and $\beta$ actin (Cell Signaling Technology, \#3700). The membrane was then visualized using an Enhanced Chemiluminescence Kit (Amersham Biosciences, Chalfont St. Giles, UK).

\section{Statistical analysis}

All results were presented as the mean \pm s.d. using GraphPad Prism 6.00 (GraphPad Software, San Diego, CA, USA) and analyzed using Student's $t$ test or ANOVA (analysis of variance). Pearson's linear correlation coefficients were used to measure the dependency of two variables. The level of significance was set at $P<0.05$.

\section{ACKNOWLEDGEMENTS}

We thank Yongkui Wang, Zhe Xing, Kai Li, and Bin Huang for providing technical support. We also thank the anonymous peer reviewers of this manuscript for their constructive comments. This work was supported by grants from National Natural Science Foundation of China (Grant Nos. 81371990, 81625015, 81601945, and
81530070) and the Program for Changjiang Scholars and Innovative Research Team in University (IRT_16R37).

\section{AUTHOR CONTRIBUTIONS}

C.L., L.L., and Z.-K.C. conducted the majority of the experiments, analyzed data, and prepared the manuscript. C.Z. conducted the surgery, Y.C. and P.L. helped with microCT analysis. H.W., Y.S., and H.Z. helped with cell culture, qPCR, western blot, and ELISA analysis. C.Z., H.F., and R.Z. provided human specimens. D.C. and X.B. supervised the project, conceived the experiments, and wrote most of the manuscript. All authors reviewed and approved the manuscript.

\section{ADDITIONAL INFORMATION}

The online version of this article (https://doi.org/10.1038/s41413-018-0041-8) contains supplementary material, which is available to authorized users.

Competing interests: The authors declare no competing interests.

\section{REFERENCES}

1. Zhen, G. et al. Inhibition of TGF-beta signaling in mesenchymal stem cells of subchondral bone attenuates osteoarthritis. Nat. Med. 19, 704-712 (2013).

2. Hootman, J. M. \& Helmick, C. G. Projections of US prevalence of arthritis and associated activity limitations. Arthritis Rheum. 54, 226-229 (2006).

3. Pereira, D. et al. The effect of osteoarthritis definition on prevalence and incidence estimates: a systematic review. Osteoarthr. Cartil. 19, 1270-1285 (2011).

4. Carr, A. J. et al. Knee replacement. Lancet 379, 1331-1340 (2012).

5. Robinson, W. H. et al. Low-grade inflammation as a key mediator of the pathogenesis of osteoarthritis. Nat. Rev. Rheumatol. 12, 580-592 (2016).

6. Chapman, K. \& Valdes, A. M. Genetic factors in OA pathogenesis. Bone 51, 258-264 (2012).

7. Chu, C. R. et al. Mechanically stimulated biomarkers signal cartilage changes over 5 years consistent with disease progression in medial knee osteoarthritis patients. J. Orthop. Res. 36, 891-897 (2018).

8. Shane, A. A. \& Loeser, R. F. Why is osteoarthritis an age-related disease? Best. Pract. Res. Clin. Rheumatol. 24, 15-26 (2010).

9. Lohmander, L. S., Gerhardsson, D. V. M., Rollof, J., Nilsson, P. M. \& Engstrom, G. Incidence of severe knee and hip osteoarthritis in relation to different measures of body mass: a population-based prospective cohort study. Ann. Rheum. Dis. 68, 490-496 (2009).

10. Chen, Y. et al. Abnormal subchondral bone remodeling and its association with articular cartilage degradation in knees of type 2 diabetes patients. Bone Res. 5, 17034 (2017).

11. Ilas, D. C., Churchman, S. M., McGonagle, D. \& Jones, E. Targeting subchondral bone mesenchymal stem cell activities for intrinsic joint repair in osteoarthritis. Future Sci. OA 3, O228 (2017).

12. Goldring, S. R. Alterations in periarticular bone and cross talk between subchondral bone and articular cartilage in osteoarthritis. Ther. Adv. Musculoskelet. Dis. 4, 249-258 (2012).

13. Lories, R. J. \& Luyten, F. P. The bone-cartilage unit in osteoarthritis. Nat. Rev. Rheumatol. 7, 43-49 (2011).

14. Cinque, M. E., Dornan, G. J., Chahla, J., Moatshe, G. \& LaPrade, R. F. High rates of osteoarthritis develop after anterior cruciate ligament surgery: an analysis of 4108 patients. Am. J. Sports Med. 46, 2011-2019 (2018).

15. Thijssen, E., van Caam, A. \& van der Kraan, P. M. Obesity and osteoarthritis, more than just wear and tear: pivotal roles for inflamed adipose tissue and dyslipidaemia in obesity-induced osteoarthritis. Rheumatology (Oxf.) 54, 588-600 (2015).

16. de Zwart, A. H. et al. Factors associated with upper leg muscle strength in knee osteoarthritis: a scoping review. J. Rehabil. Med. 50, 140-150 (2018).

17. Tanamas, S. K. et al. Bone marrow lesions in people with knee osteoarthritis predict progression of disease and joint replacement: a longitudinal study. Rheumatology (Oxf.) 49, 2413-2419 (2010).

18. Loewith, R. \& Hall, M. N. Target of rapamycin (TOR) in nutrient signaling and growth control. Genetics 189, 1177-1201 (2011).

19. Reiling, J. H. \& Sabatini, D. M. Stress and mTORture signaling. Oncogene 25, 6373-6383 (2006).

20. Wullschleger, S., Loewith, R. \& Hall, M. N. TOR signaling in growth and metabolism. Cell 124, 471-484 (2006).

21. Jiang, M., Fu, X., Yang, H., Long, F. \& Chen, J. mTORC1 signaling promotes limb bud cell growth and chondrogenesis. J. Cell. Biochem. 118, 748-753 (2017).

22. Yan, B. et al. mTORC1 regulates PTHrP to coordinate chondrocyte growth, proliferation and differentiation. Nat. Commun. 7, 11151 (2016). 
23. Zhang, $H$. et al. mTORC1 activation downregulates FGFR3 and PTH/PTHrP receptor in articular chondrocytes to initiate osteoarthritis. Osteoarthr. Cartil. 25, 952-963 (2017).

24. Zhang, Y. et al. mTORC1 Inhibits NF-kappaB/NFATc1 Signaling and prevents osteoclast precursor differentiation, in vitro and in mice. J. Bone Miner. Res. 32, 1829-1840 (2017).

25. Huang, B. et al. mTORC1 prevents preosteoblast differentiation through the Notch signaling pathway. PLoS Genet. 11, e1005426 (2015).

26. Dai, Q. et al. mTOR/Raptor signaling is critical for skeletogenesis in mice through the regulation of Runx2 expression. Cell Death Differ. 24, 1886-1899 (2017).

27. Jo, D. Y., Rafii, S., Hamada, T. \& Moore, M. A. Chemotaxis of primitive hematopoietic cells in response to stromal cell-derived factor-1. J. Clin. Invest. 105, 101-111 (2000)

28. Wei, L. et al. Stimulation of chondrocyte hypertrophy by chemokine stromal cellderived factor 1 in the chondro-osseous junction during endochondral bone formation. Dev. Biol. 341, 236-245 (2010).

29. Kanbe, K., Takagishi, K. \& Chen, Q. Stimulation of matrix metalloprotease 3 release from human chondrocytes by the interaction of stromal cell-derived factor 1 and CXC chemokine receptor 4. Arthritis Rheum. 46, 130-137 (2002).

30. Wei, F. et al. Attenuation of osteoarthritis via blockade of the SDF-1/ CXCR4 signaling pathway. Arthritis Res. Ther. 14, R177 (2012).

31. Tchetina, E. V. et al. Differences in mammalian target of rapamycin gene expression in the peripheral blood and articular cartilages of osteoarthritic patients and disease activity. Arthritis 2013, 1-14 (2013).

32. Zhang, Y. et al. Cartilage-specific deletion of mTOR upregulates autophagy and protects mice from osteoarthritis. Ann. Rheum. Dis. 74, 1432-1440 (2015).

33. Lorenz, J. \& Grassel, S. Experimental osteoarthritis models in mice. Methods Mol. Biol. 1194, 401-419 (2014).

34. Chen, Y. et al. Bone turnover and articular cartilage differences localized to subchondral cysts in knees with advanced osteoarthritis. Osteoarthr. Cartil. 23, 2174-2183 (2015).

35. Zhen, G. \& Cao, X. Targeting TGFbeta signaling in subchondral bone and articular cartilage homeostasis. Trends Pharmacol. Sci. 35, 227-236 (2014).

36. Saito, T. et al. Transcriptional regulation of endochondral ossification by HIF2alpha during skeletal growth and osteoarthritis development. Nat. Med. 16, 678-686 (2010)

37. Hugle, T. \& Geurts, J. What drives osteoarthritis?-Synovial versus subchondral bone pathology. Rheumatology (Oxf.) 56, 1461-1471 (2017).

38. Zuo, Q. et al. Characterization of nano-structural and nano-mechanical properties of osteoarthritic subchondral bone. BMC Musculoskelet. Disord. 17, 367 (2016).

39. Reece, D. S., Thote, T., Lin, A., Willett, N. J. \& Guldberg, R. E. Contrast enhanced muCT imaging of early articular changes in a pre-clinical model of osteoarthritis. Osteoarthr. Cartil. 26, 118-127 (2018).

40. Li, G. et al. Subchondral bone in osteoarthritis: insight into risk factors and microstructural changes. Arthritis Res. Ther. 15, 223 (2013).

41. Burr, D. B. \& Radin, E. L. Microfractures and microcracks in subchondral bone: are they relevant to osteoarthrosis? Rheum. Dis. Clin. N. Am. 29, 675-685 (2003).

42. Stein, V. et al. Pattern of joint damage in persons with knee osteoarthritis and concomitant ACL tears. Rheumatol. Int. 32, 1197-1208 (2012).
43. Goldring, M. B. \& Goldring, S. R. Articular cartilage and subchondral bone in the pathogenesis of osteoarthritis. Ann. NY Acad. Sci. 1192, 230-237 (2010).

44. Fang, F. et al. Neural crest-specific TSC1 deletion in mice leads to sclerotic craniofacial bone lesion. J. Bone Miner. Res. 30, 1195-1205 (2015).

45. Thomas, N. P. et al. Attenuation of cartilage pathogenesis in post-traumatic osteoarthritis (PTOA) in mice by blocking the stromal derived factor 1 receptor (CXCR4) with the specific inhibitor, AMD3100. J. Orthop. Res. 33, 1071-1078 (2015).

46. Dong, Y. et al. Inhibition of SDF-1a/CXCR4 signalling in subchondral bone attenuates post-traumatic osteoarthritis. Int. J. Mol. Sci. 17, 943 (2016).

47. Wang, S. X., Laverty, S., Dumitriu, M., Plaas, A. \& Grynpas, M. D. The effects of glucosamine hydrochloride on subchondral bone changes in an animal model of osteoarthritis. Arthritis Rheum. 56, 1537-1548 (2007).

48. Glasson, S. S., Blanchet, T. J. \& Morris, E. A. The surgical destabilization of the medial meniscus (DMM) model of osteoarthritis in the 129/SvEv mouse. Osteoarthr. Cartil. 15, 1061-1069 (2007).

49. Pritzker, K. P. et al. Osteoarthritis cartilage histopathology: grading and staging. Osteoarthr. Cartil. 14, 13-29 (2006).

50. Chen, J. et al. Osx-Cre targets multiple cell types besides osteoblast lineage in postnatal mice. PLoS ONE 9, e85161 (2014).

51. Rodda, S. J. \& McMahon, A. P. Distinct roles for Hedgehog and canonical Wnt signaling in specification, differentiation and maintenance of osteoblast progenitors. Development 133, 3231-3244 (2006).

52. Bucan, M. in Brenner's Encyclopedia of Genetics 2nd edn (eds Maloy, S. \& Hughes, K.) 486-488 (Academic Press, San Diego, 2013).

53. Glasson, S. S., Chambers, M. G., Van Den Berg, W. B. \& Little, C. B. The OARSI histopathology initiative-recommendations for histological assessments of osteoarthritis in the mouse. Osteoarthr Cartil. 18, S17-S23 (2010).

54. Hou, C. et al. The role of microRNA-381 in chondrogenesis and interleukin-1-beta induced chondrocyte responses. Cell. Physiol. Biochem. 36, 1753-1766 (2015).

55. Tropel, P. et al. Isolation and characterisation of mesenchymal stem cells from adult mouse bone marrow. Exp. Cell Res. 295, 395-406 (2004).

56. Bellows, C. G. \& Aubin, J. E. Determination of numbers of osteoprogenitors present in isolated fetal rat calvaria cells in vitro. Dev. Biol. 133, 8-13 (1989).

Open Access This article is licensed under a Creative Commons Attribution 4.0 International License, which permits use, sharing, adaptation, distribution and reproduction in any medium or format, as long as you give appropriate credit to the original author(s) and the source, provide a link to the Creative Commons license, and indicate if changes were made. The images or other third party material in this article are included in the article's Creative Commons license, unless indicated otherwise in a credit line to the material. If material is not included in the article's Creative Commons license and your intended use is not permitted by statutory regulation or exceeds the permitted use, you will need to obtain permission directly from the copyright holder. To view a copy of this license, visit http://creativecommons. org/licenses/by/4.0/.

(c) The Author(s) 2019 